\title{
Novel Pliable Electrodes for Flexible Electrochemical Energy Storage Devices: Recent Progress and Challenges
}

\author{
Muhammad Yousaf, Hao Tian H. Shi, Yunsong Wang, Yijun \\ Chen, Zhimin Ma, Anyuan Cao, Hani E. Naguib, \\ and Ray P.S. Han
}

Version Post-print/accepted manuscript

Citation Yousaf, M., Shi, H. T. H., Wang, Y., Chen, Y., Ma, Z., Cao, A., ... \&

(published version) Han, R. P. (2016). Novel pliable electrodes for flexible electrochemical energy storage devices: recent progress and challenges. Advanced Energy Materials, 6(17).

Publisher's Statement This is the peer reviewed version of the following article: Yousaf, M., Shi, H. T. H., Wang, Y., Chen, Y., Ma, Z., Cao, A., ... \& Han, R. P. (2016). Novel pliable electrodes for flexible electrochemical energy storage devices: recent progress and challenges. Advanced Energy Materials, 6(17).

which has been published in final form at 10.1002/aenm.201600490 This article may be used for non-commercial purposes in accordance with Wiley Terms and Conditions for Self-Archiving.

How to cite TSpace items

Always cite the published version, so the author(s) will receive recognition through services that track citation counts, e.g. Scopus. If you need to cite the page number of the author manuscript from TSpace because you cannot access the published version, then cite the TSpace version in addition to the published version using the permanent URI (handle) found on the record page.

This article was made openly accessible by $U$ of $T$ Faculty.

Please tell us how this access benefits you. Your story matters. 


\title{
Novel Pliable Electrodes for Flexible Electrochemical Energy Storage Devices: Recent Progress and Challenges
}

\author{
Muhammad Yousaf, Hao Tian H. Shi, Yunsong Wang, Yijun Chen, Zhimin Ma, \\ Anyuan Cao, Hani E. Naguib, and Ray P.S. Han*
}

With wearable electronics rapidly coming into fashion, research into flexible energy storage devices and in particular, their pliable electrodes is attracting a lot of attention. Pliable electrodes are usually fabricated by intercalating an active material in a flexible matrix with superior mechano-electrical properties, and can be grouped either as substrate-supported or free-standing. Further, depending on their mode of deformation the electrodes can be labeled as bendable, compressible or stretchable. This Progress Report highlights the recent progress and challenges in the design and fabrication of pliable electrodes for constructing flexible lithium ion batteries and flexible supercapacitors. We also present 2 pliable core-shell structure electrodes fabricated from: (a) carbon nanotube sponge embedded with $\mathrm{MoS}_{2}$ nanoparticles and (b) electrospun polyethylene terephthalate fibers impregnated with graphene nanoplatelets.

\section{Introduction}

The advent and rapid development of integrated wearable electronic devices, wireless sensors, roll-up displays, pliable smartcards, electronic skins, flexible smartphones, portable and implantable medical devices, etc. is driving the huge interest surge in flexible energy storage devices. ${ }^{[1]}$ Many electronic companies have launched flexible concepts and products such as Nokia Morph, Philip Morph,

KEYWORDS: Flexible Energy Storage Devices, Flexible Lithium Ion Battery, Flexible Supercapacitors, Substrate Supported Pliable Electrodes, Free Standing Pliable Electrodes, CNT@MoS 2 Pliable Electrodes, PET@GnPs Pliable Electrodes

M. Yousaf, Y. Wang, Y. Chen, Z. Ma, Prof. A. Cao, Prof. R.P.S. Han*

Department of Materials Science \& Engineering, Peking University, Beijing, China 100871

H.T.H. Shi, Prof. H.E Naguib

Department of Mechanical \& Industrial Engineering,

University of Toronto, Toronto, Canada M5S3G8

Prof. H.E Naguib

Department of Materials Science \& Engineering, University

of Toronto, Toronto, Canada M5S1A1

*Email: ray-han@pku.edu.cn
iPhone ProCare, Filip Fluid, LG G Flex, Intel Jarvis, Samsung Galaxy Gear, Google Glass, etc. ${ }^{[2]}$ The most frequent challenge that is bottlenecking the practical implementation of flexible systems is the availability of flexible and reliable power sources with a high energy density, long cycle life and excellent rate capability. To be acceptable for use, these electronic devices require a small, flexible and lightweight energy storage system that is robust under bending, twisting, compressing and stretching deformations without compromising their functions and performance. Flexible energy storage systems such as flexible alkaline batteries, ${ }^{[3]}$ flexible zinc carbon batteries ${ }^{[4]}$ all-polymer batteries, ${ }^{[5]}$ polymer lithium-metal batteries, ${ }^{[6]}$ flexible rechargeable lithium ion batteries $(\mathrm{LIBs})^{[7]}$ and flexible supercapacitors $(\mathrm{SCs})^{[8]}$ have been explored and investigated. Among these, flexible LIBs and SCs are very promising due to their higher energy and power density, longer life and environmental benignity. ${ }^{[9]}$

Flexible LIBs and SCs have the same charge storage mechanism as conventional LIBs or SCs and are popularly used in electronics, electric vehicles (EV) and hybrid electric vehicles (HEV). ${ }^{[10]}$ Under external loadings, battery materials undergo mechanical deformations including bending, twisting, compressing and as well stretching, which can seriously affect the inherent structure of the electrode materials along with electrode/electrolyte transport properties. ${ }^{[11]}$ Recently, good progress has been made in the development of core components of flexible LIBs and SCs (electrode materials, electrolytes and current collector). Nevertheless, the big challenges in procuring flexible energy storage devices are the fabrication of pliable electrode materials with high surface area, high specific capacity and good conductivity, selection of flexible and bendable current collectors with excellent mechanical properties (low density, roughened surface and tunable thickness), and solid state electrolyte with high mechanical properties and ionic conductivity. A good 
structural design can help to mitigate some of these obstacles to achieve some degrees of extended flexibility.

The most important component of flexible LIBs and SCs are the pliable electrodes, which possess advantages such as flexibility, lightweight, high energy density and high rate performance over rigid electrodes. It is not difficult to integrate pliable electrodes into single thin electrode material to yield a higher energy density, which is in contrast to rigid electrodes that consist of a mixture of active material, conductive additive and binders casted onto heavy metal current collectors. Also, due to superior electronic and ionic conductivities of carbon based flexible support, pliable electrodes can exhibit comparable rate performances to rigid electrodes. However, they possess some serious disadvantages in terms of cyclic stability, safety and large scale production. Their mechanical and electrochemical stabilities are lower as a result of each successive and varying mechanical deformation. ${ }^{[12]}$

Our report targets the recent progress on flexible energy storage devices, including flexible LIBs and SCs. In first part, we highlight the challenges associated with flexible LIBs and SCs and then, we discuss the various fabrication processes for electrode materials in flexible LIBs based on different structure, deformation and design. In second part we present some recent developments inflexible SCs with the focus on 3-dimensional (3D) free standing hydrogels, 2D free standing paper-based and flexible substrate supported electrodes. We have also included in the discussion our recent progress work on flexible LIBs and SCs. It should be noted that most review articles focus on the 2D planer, 3D bulky structure with bendable or compressible deformations in LIBs or SCs, which are used in flexible electronics but are constrained in their potential applications for future electronic devices that require omnidirectional flexibility. ${ }^{[7,9 f, 13]}$ Some recent articles have reported on fiber-shaped LIBs or SCs that fulfill the requirements of omni-directional flexibility. ${ }^{[14]}$ In our review, we summarized and provided a comprehensive overview of the 2D planer, 3D bulky structures and fibershaped structure for LIBs and SCs.

A schematic sketch of flexible LIBs and SCs with different designs and structures along with the types of electrode used in their fabrication is given in Figure 1.

\section{Challenges Associated with Flexible LIBs and SCs}

In flexible LIBs and SCs, the term flexibility implies the ability of being bended, but a more extensive meaning of the term includes the additional ability of being twisted, compressed and stretched. These extra modes of deformation not only generate more challenges for the electrode materials, current collectors and electrolytes but also, pose greater difficulties in the manufacture of flexible LIBs and SCs. In this section we introduce the main

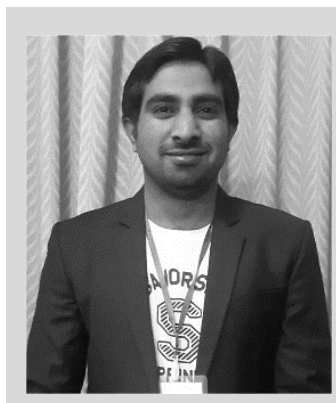

Muhammad Yousaf is pursuing his Ph.D. in Materials Science and Engineering under the supervision of Professor Ray P.S. Han from Peking University. He completed his M.S. degree in 2013 in Solid State Physics from Punjab University, Lahore and undergraduate degree in 2010 in Applied Physics from University of Engineering and Technology, Lahore. His research includes layered transition metal dichalcogenides based composites and their application in flexible energy storage devices.

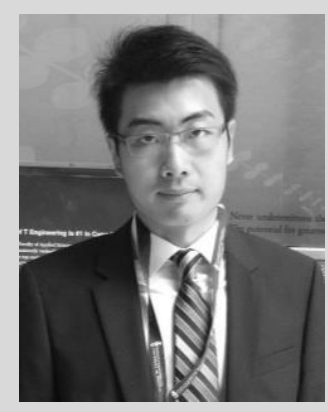

HaoTian Harvey Shi is completing his Master's of Applied Science studies at the University of Toronto, under the supervision of Prof. Hani E. Naguib from the Department of Mechanical and Industrial Engineering. He obtained his Bachelor's degree from the Department of Engineering Science at the University of Toronto in 2014. His research focus is mainly on the fabrication and optimization of flexible and lightweight thin-film coreshell structured electrochemical capacitors electrodes for energy storage applications

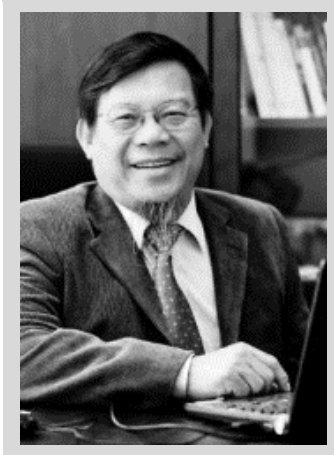

Ray P.S. Han is a Changjiang Professor of Materials Science and Engineering and Assistant Dean of Engineering at Peking University, Beijing China. His research is focused on flexible electrodes for fabricating flexible Li-ion batteries and supercapacitors, harvesting ambient nano-mesoscale energies for self-powered sensors and devices, and development of nano-micro microfluidic devices.

challenges associated with flexible LIBs and SCs and the requirements of their constituents components.

The main component of LIBs and SCs is their electrodes and it is very difficult to manufacture pliable electrode structure, due mainly to the lack of appropriate materials that are not only mechanically flexible but also, possessing a high conductivity and a good electrochemical performance. In conventional LIBs and SCs, the electrode 


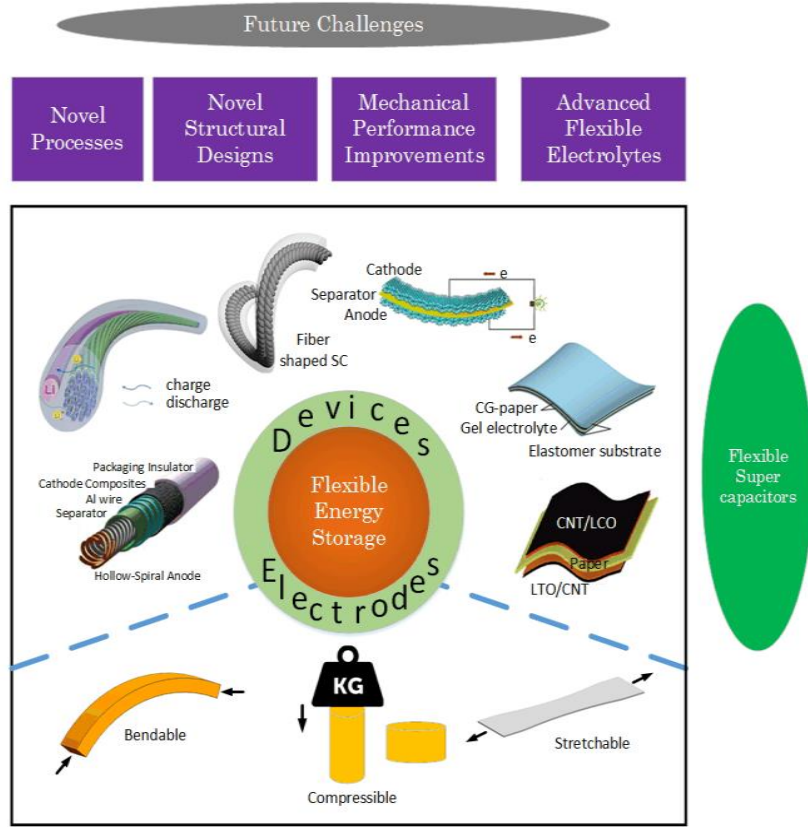

Figure 1. Proposed flexible energy storage devices and the types of electrode used in their fabrication.

materials are fabricated using a slurry-casting method in which, active materials in powder form, binders and conductive additives (mostly carbon black or CNT) are mixed together and casted onto metal current collectors. ${ }^{[9 f, 15]}$ The binders provide an interconnection to the active materials, conductive additives and current collectors, while the conductive additives provide an electronic pathway between electrode materials and current collectors. The two materials also have some significant drawbacks; they are less likely to contribute to the charge storage phenomenon and they make up about $20 \%$ of the overall mass and thus, generate a decrease in the gravimetric/volumetric energy densities. Further, active materials such as activated carbon, graphite, $\mathrm{LiCoO}_{2}$, silicon ( $\mathrm{Si}$ ), metal oxides and other inorganic materials are intrinsically brittle and incapable of undergoing any finite deformation associated with bending, twisting, compressing or stretching. Hence, the development of highly flexible and mechanically strong electrodes with flexible structures is required to improve the electrochemical performance of flexible LIBs and SCs. This may be accomplished by eliminating the binders and conductive additives from the active electrode material and growing or pasting them directly on to the flexible substrates. The current collectors are used to provide structural support to the electrode material and the electrical pathway between the electrode material and the outer circuit of the cell. Current collectors suffer from 3 major problems: (1) in traditional LIBs the current collectors are mostly metallic - aluminum (Al) for the cathode and copper $(\mathrm{Cu})$ for the anode and they collectively, account for between $15 \%$ and $50 \%$, respectively, of the overall mass of the cathode and anode, ${ }^{[16]}$ resulting in lower the energy densities of the LIBs. (2) Due to the flat and smooth surface of the metal current collectors, a very weak adhesion and limited contact between the electrode materials and the current collector takes place. During the cycling process, volumetric changes occur in the electrode materials, producing gaps at the interface of the electrode materials and the current collectors, and this result in a loss of capacity, stability and a low rate capability. For flexible batteries, the issue becomes more severe during mechanical deformations (bending, twisting, compressing and stretching and their combinations), leading to a delamination of the electrode materials. (3) during the long term cycling process, the metal current collectors remain in a corrosive environment for a prolonged duration and this make them susceptible to pitting corrosion and cracking in the $\mathrm{Al}$ and $\mathrm{Cu}$ respectively, which in turn, results in a loss of capacity and poor rate performance. The energy density and stability can be enhanced by replacing the heavy and rigid current collectors with flexible and lightweight versions such as graphene or CNT papers. ${ }^{[17]}$

In traditional LIBs and SCs, the used liquid electrolyte is electronically insulator and acts mainly as a medium to allow ions to diffuse between cathode and anode. However, in comparison to conventional LIBs and SCs, the use of liquid electrolyte in flexible devices can be quite challenging due to mechanical deformations. Further, the mechanical deformation of flexible devices could lead to a short-circuiting that could result in an explosion. These safety issues have compelled the scientific community to look for solid state electrolytes, which can also, improve the cell life. However, the availability of solid state electrolytes have lowered the room temperature ionic conductivity $\left(\sim 10^{-4} \mathrm{~S} \mathrm{~cm}^{-1}\right)$, which could seriously undermine the charge transport. In addition, these solid state electrolytes suffer from poor interfacial contact with active materials, leading to a poor performance. The development of polymer or gel based solid electrolytes with enhanced ionic conductivity and mechanical properties is therefore, urgently required.

Integrating and packaging of each flexible component such as the electrode materials, current collector and electrolytes into a single device is highly sought. Flexible packaging materials that can bend and stretch in sync with other components of a flexible device to prevent the electrolyte from escaping are needed. ${ }^{[11]}$ In addition to the flexibility issue of these power sources, there are 2 lifestyle demands that need to be met: (1) these electrochemical devices have to be ultrathin and ultralight compared to traditional LIBs or SCs in order to qualify as an ultrathin wearable electronic device and (2) they should be available 
in various shapes to conform to curve surfaces like the human skin. This can be achieved through a stretchable deformation.

\section{Electrode Materials for Flexible LIBs}

The electrochemical performance of LIBs is largely dependent on the active material and electrode structure with the configuration of pliable electrodes affecting the full battery design. Flexible LIBs can be categorized into 3 types on the basis of their deformation characteristics, structure and dimension and the electrodes materials: (1) bendable electrodes, (2) compressible electrodes and (3) stretchable electrodes. In the following sections we will describe each of the electrode system used in a full battery design.

\subsection{Bendable Electrodes (BEs)}

BEs are designed to work under a bending deformation and all its components; the substrate, current collector and active material continue to perform under a bending deformation. BEs can be classified as one of 2 types: 1) substrate supported BEs, in which the active material is deposited or casted onto a flexible substrate attached to the current collector and 2) free standing BEs, where the active materials are mixed with mechanically resilient and highlyconductive materials. The 2 electrode types have their advantages and disadvantages. For example, in the substrate supported BEs, the mechanical strength and flexibility is provided by the substrate used (conventional paper or textile), which could be highly elastic and hence, good for fabricating flexible storage devices. On the other hand, they also have disadvantages in holding the active materials on its binder. Binders are electrically insulators that result in an overall decrease in the energy density of the battery. Further, during deformations the active materials may delaminate from the current collector due the limited contact area between the active materials and the current collector. The free standing BEs have high $\mathrm{Li}$ storage capacity and energy density due to the absence of a substrate and binder, but they suffer from limited mechanical strength and currently, they are still evolving from laboratory-level investigation.

\subsubsection{Substrate Supported BEs}

The strength and flexibility of this electrode is strongly dependent on the type of substrate employed. Substrates can be divided into 2 types: conductive and non-conductive substrates. Carbon based films such as CNT and graphene are popularly used as a conductive substrate, which also works as a current collector. On the other hand, conventional paper, textile, polymer, plastic film etc. are frequently used as a non-conductive substrate. ${ }^{[13]} \mathrm{A}$ suitable flexible substrate should possess good mechanical properties (yield strength and elastic deformation), strong bonding with the active materials, adequate stability with the electrolyte and high electronic and ionic conductivities. The current collector is an essential component of a battery and the characteristics of the substrate determine whether a current collector is required to be fabricated as a part of the electrode preparation process. In case of a conductive substrate, the current collector is not needed as the substrate can act as a current collector, whereas for a nonconductive substrate, an additional conductive element should be deposited on it to act as a current collector.

Non-conductive substrates. Non-conductive substrates are very flexible as they possess large elastic limits but still, they are an electrical insulator. To make them conductive, a coating of a conductive layer, usually, a carbon based material such as CNT or graphene can be used. Methods such as a vacuum filtration, spraying and direct coating is used to make a non-conductive substrate acts as a current collector. Cellulose paper, textiles and polymers etc. are popular as a non-conductive substrate due to their high flexibility and adequate mechanical strength. Cellulose paper for example, is lightweight, lowcost, porous, easily processable, recoverable and environmentally friendly. It has attracted much attention in lightweight and flexible electronic devices. ${ }^{[18]}$ There are two strategies to make a paper conductive: coating with a conductive layer and fabricating a composite paper by adding conductive additives to the cellulose. A nanocomposite of cellulose paper with CNT and graphene has also been developed and used as BEs for a flexible LIB. However, a $\mathrm{Li}$ foil is used as a counter electrode, which is deposited on the metal foil current collector but it can easily break under bending. ${ }^{[19]}$ Cui et al. ${ }^{[20]}$ demonstrated that a commercial paper can be easily turned conductive with a conformal coating of a SWCNT ink via the Meyer rod coating method, which can be used as metal free current collector in an LIB (Figure 2a, b). They further showed that the conductive paper can be bent up to a 100 times without noticeable structural degradation while still retaining a good electrical conductivity. They also claimed that the adhesion of film on the paper is better than plastic and the resistance of CNT paper is highly dependent on the thickness of the CNT film (Figure 2c-e). To evaluate the electrochemical performance of the battery, a slurries of cathode $\left(\mathrm{LiMn}_{2} \mathrm{O}_{4}(\mathrm{LMO})\right)$ and anode materials $\left(\mathrm{Li}_{4} \mathrm{Ti}_{5} \mathrm{O}_{12}\right.$ (LTO)) were coated onto the conductive paper and with a half cell and a full cell it performed well (Figure 2f-h). In a similar way, a simple dipping/soaking and drying method was reported in which a polycellulose paper or a Kimwipes substrate is made conductive with a CNT/MWCNT ink solution and used as a flexible/foldable current collector for LIB. $^{[21]}$

Textile is also an ideal non-conductive substrate that is lightweight, flexible, has more surface porosity than paper and can bear higher temperatures up to $200-300{ }^{\circ} \mathrm{C}$, which 

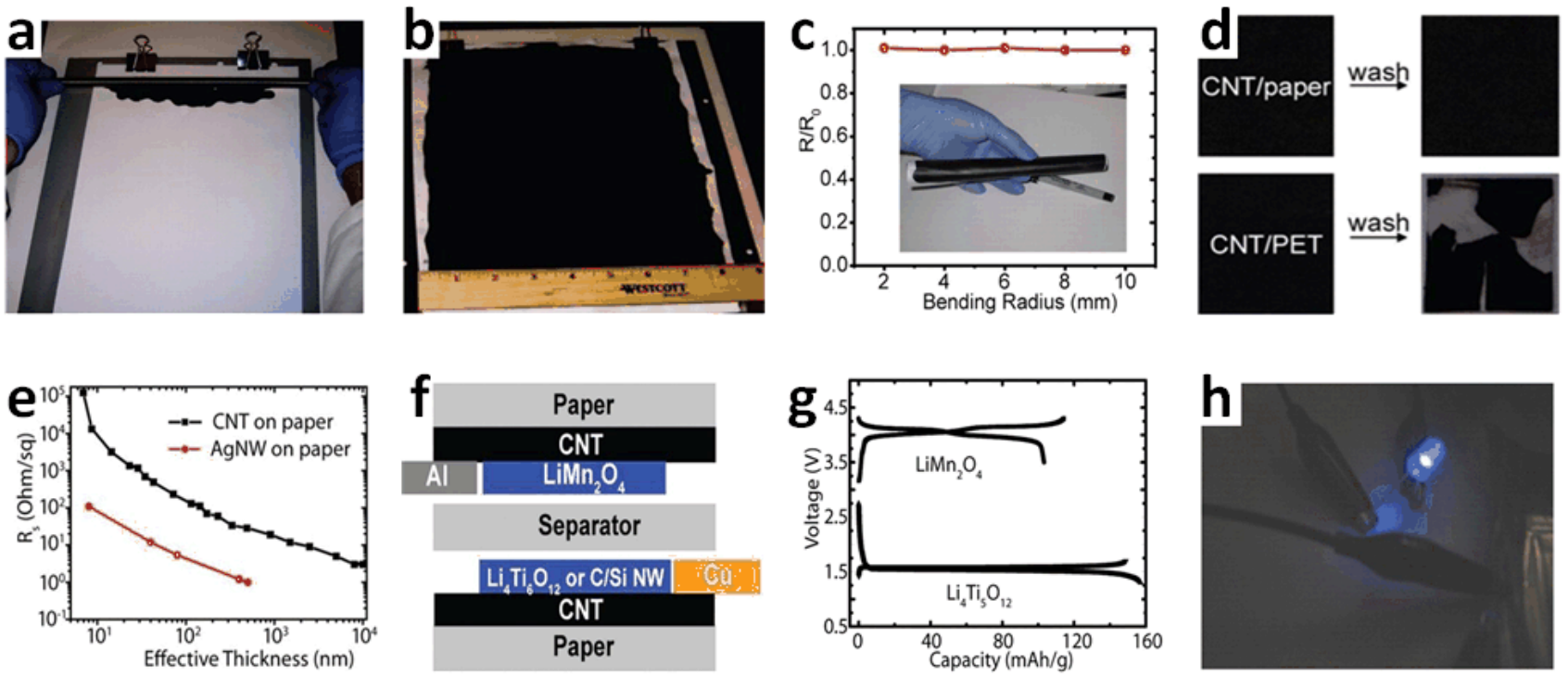

Figure 2. (a, b) Conformal coating of CNTs ink on paper by Meyer rod. (c) Sheet resistance changes after bending conductive paper into different radii. (d) The comparison of film peeling after washing surfactants. (e) Sheet resistances of conductive paper based on CNTs with various thicknesses. (f) Schematic illustration of the conductive paper battery configuration. (g) Galvanostatic charging/ discharging curves of LMO nanorod cathode (3.5- 4.3 V) and LTO nano powder anode $(1.3-1.7 \mathrm{~V})$ half-cells with conductive paper current collectors at $\mathrm{C} / 5$. (h) A full battery used to light a blue LED. Reproduced with permission. ${ }^{[20]}$ Copyright 2009, PNAS.

makes it highly desirable for many applications. ${ }^{[13]}$ The surface chemistry of a textile makes it relatively easy to adhere an active material to allow for fast ion migrations. Therefore, it is obvious that a textile possesses tremendous potential for applications in flexible electronic and energy storage devices, ${ }^{[22]}$ but still, it is an electrical insulator and hence, cannot be directly used as an electrode. A porous polyester fiber textile can be made conductive by soaking into a CNT ink and after drying be used as a current collector for anode and cathode; further, it is reported that the active material mass loading of textile current collector is about 8 times higher compared to a metal current collector. ${ }^{[23]}$ With the same active mass loading, the textile current collector showed better performance than that of flat metal current collector and this was attributed to a reduced contact impedance due to the better contact between the active material and current collector. ${ }^{[23]}$

Conductive substrate. Conductive substrates have also been used to hold active materials such as CNT and graphene films. The CNT or graphene is not homogenously mixed with the active materials but rather, are laminated together for use as a current collector. As in traditional LIBs, the current collectors are usually made of heavy and smooth metals and have weak adhesion with the active material and this reduces the overall energy density and stability of the cell. CNT and graphene film based current collectors are flexible, lighter and rough, which facilitates interface contacts and weight reduction. They have strong mechanical properties due to their 1D/2D nanostructure and with the small radius of curvature, they make very good BEs.

A conductive substrate fabricated from a Xerox paper ${ }^{[24]}$ was used as a current collector for both anode and cathode of a flexible LIB. The Xerox paper worked as both a separator and a mechanical support with the highly conductive free standing CNT film acting as current collectors. The active materials, $\mathrm{LTO}$ as anode and $\mathrm{LiCoO}_{2}$ (LCO) as cathode were blade-casted onto the CNT film. For a full battery design the anode (LTO/CNT) and cathode (LCO/CNT) materials were coated on both sides of the substrate (Xerox paper) by a lamination process and encapsulated with a $10 \mu \mathrm{m}$-polydimethylsiloxane (PDMS) coating. After packaging, the full cell has thickness of about $300 \mathrm{~mm}$ and is highly flexible (bent downwards to $<6 \mathrm{~mm}$ radius 50 times). In a similar way, a CNT film has been drawn from super-aligned CNT (SACNT) arrays to act as a current collector for an LIB. ${ }^{[17]}$ The result is a very thin, nicely flexible and light weight collector compared to metal flat current collectors. Further, it possesses strong adhesion, good wetting, high mechanical durability and low contact resistance. ${ }^{[17]}$

Recently, a new electrode design was demonstrated, in which the CNT film was prepared by a spray coating method to function as a highly flexible current collector (Figure 3a). For the electrode fabrication, a nonwoven fibrous membrane was dipped into slurries of anode (LTO) and cathode (LCO) active materials and then, mechanically pressed onto a free standing CNT current collector (Figure 
3b, c) ${ }^{[25]}$ The full battery (Figure 3d) showed a 3-4 times higher performance in terms of areal capacity compared to similar reports using the same LTO/LCO active materials. ${ }^{[26]}$ Additionally, the battery was mechanically flexed to varying bending radii for different electrochemical cycles and no any significant drop in areal capacity was observed after a 100-time flexing (Figure 3e). The higher performance can be attributed to the highly flexible and conductive membrane, which supports the active materials and inhibits it from cracking during the flexing. Further, with the conductive CNT current collector the thickness of inactive components in the flexible LIB can be kept to a minimum.
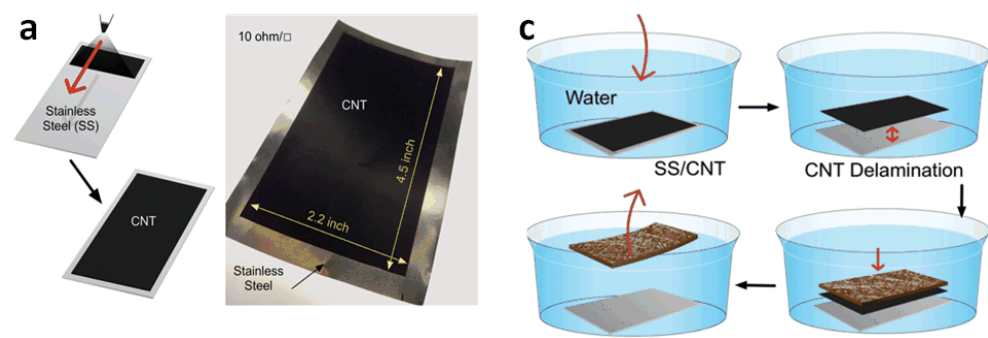

CNT Transfer on Anode/Cathode
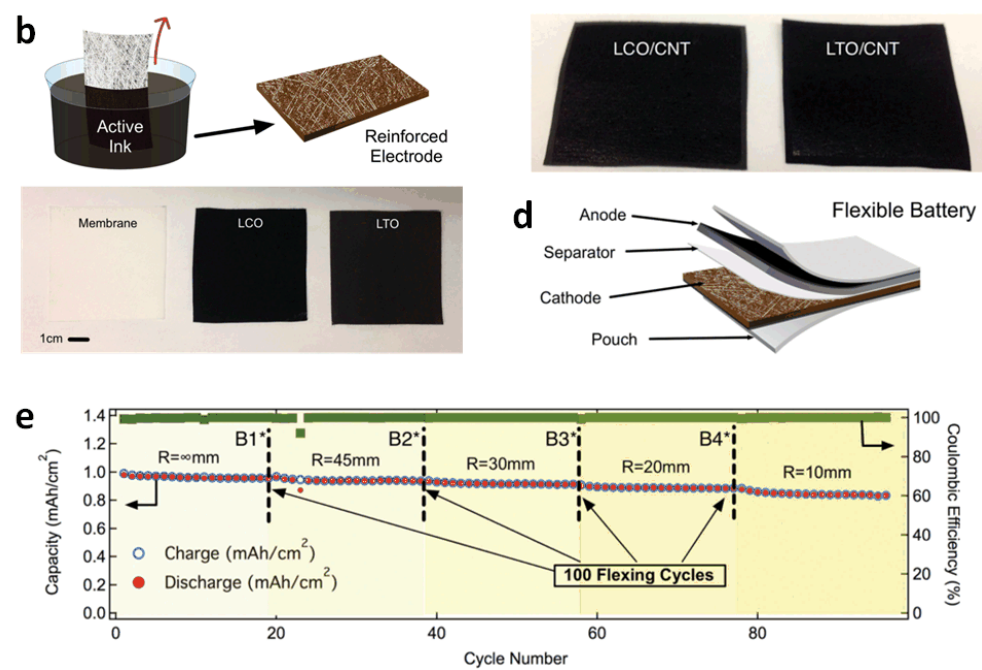

Figure 3. (a) Spray coating carbon nanotube (CNT) ink on top of stainless steel (SS). (b) Embedding the electrochemically active inks ("LCO" and "LTO" inks) within the supporting membrane by dipping inside an ink bath. (c) Schematic of the flexible battery laminated within aluminum-lined pouch. (d) Schematic of the flexible battery laminated within aluminum-lined pouch. (e) Effect on areal capacity $\left(\mathrm{mAhcm}^{-2}\right)$ of battery flexed to different radii on different electrochemical cycles. Reproduced with permission. ${ }^{[25]}$ Copyright 2015, John Wiley \& Sons, Inc.

Besides CNT films, graphene films or papers have also been used as conductive substrate or current collector for flexible LIBs. ${ }^{[27]}$ For instance, Shi et al. ${ }^{[27]}$ fabricated a highly conductive and flexible graphene paper to use as current collectors for both anode and cathode. The graphene paper was made by a filtration method and the active material of anode (LTO) and cathode $\left(\mathrm{LiFePO}_{4}\right.$ (LFP)) were deposited on the surface of the graphene paper another filtration method. ${ }^{[27 b]}$ A full cell battery was state, and the result showed superior electrochemical performance, high gravimetric/volume energy density and ood flexibility/bendability of the graphene paper.

In summary, CNT or graphene paper based current collectors are very flexible, light and thin and have wide potential applications in flexible/bendable energy storage devices. The interconnected porous channels in the CNT thin films facilitate a very fast movement of charges and the rough surface provides a large surface area for the deposition of the active materials. Also, the CNT and graphene thin film are chemically inert, which ensure a good stability of the CNT or graphene based current collectors. These highly flexible batteries are promising for use in the next generation wearable electronics.

\subsubsection{Free Standing Films for BEs}

Free standing monolithic films. The use of CNT or graphene films as both current collector and active material allows for the development of ultrathin, light and flexible LIBs. Many reports have been published for the fabrication of CNT and graphene films. ${ }^{[28]}$ Most of the films are fabricated by the vacuum filtration and CVD process. Generally, CNT or graphene film based electrodes have low mechanical strength and lack flexibility. These films also have low efficiency which prevent them for use in practical applications and thus, have been confined to mostly laboratory research. Morris et al. ${ }^{[28 \mathrm{a}]}$ studied the $\mathrm{Li}^{+}$storage capacity based on single SWCNT bucky paper and Liu et al. ${ }^{[28 \mathrm{~b}]}$ provided a comparative study among the SWCNT, DWCNT (double walled CNT) and MWCNT (multiwalled CNT) films fabricated by the vacuum filtration method in terms of their $\mathrm{Li}^{+}$storage. MWCNT film based anode showed better performance than either SWCNT or DWCNT due to its better mechanical stability after a series of electrochemical tests. In order to further increase the flexibility and mechanical strength of flexible LIB, Sun et al. ${ }^{[28 c]}$ fabricated highly flexible porous CNT film on Celgard 3500 polypropylene separator by vacuum filtration method and used as anode directly. The enhanced flexibility and mechanical strength of as prepared electrode is due to the flexibility of separator 
and its presence in the electrode does not decrease the energy density of LIB as it is essential component of battery. In the vacuum filtration method, the surfactant is an essential element for the dispersion of CNT in the solvent even after washing. As it is electrical insulator and decrease the rate performance of battery. In addition, most of paper electrodes obtained by the vacuum filtration approach have disadvantages in terms of dimensional stability and flexibility for the reason that they are formed by an aggregation of physically-stacked NPs layers. ${ }^{[28 c]}$

CVD is another approach to grow the CNT film to increase the flexibility and mechanical strength of electrodes and hence, the performance of the LIB. Chen et $a l^{[28 \mathrm{~d}]}$ synthesized CNT film integrated with a highly conductive carbon layer (CL) on conductive and nonconductive substrates by the direct CVD method and used as an anode for the LIB. The resulting composite (CNT/CL) was flexible and lightweight, and showed a high reversible capacity $\left(572 \mathrm{mAhg}^{-1}\right.$ at $0.2 \mathrm{mAcm}^{-2}$ after 100 cycles). Besides CNT, a composite of aligned CNT (ACNT) array with conducting polymer was also used to fabricate pliable electrodes for LIB. ${ }^{[28 \mathrm{e}]}$ The ACNT film is better than the SWCNT film in several aspects: high surface area, high order and excellent mechanical properties. Recently, the effect of heat treatment on the performance of a flexible LIB was proposed. ${ }^{[28 f]}$ The CNT film was fabricated via CVD and direct spinning for use as an anode of a flexible LIB, and the heat treated CNT film exhibited better electrochemical performance than the untreated one due to the high crystallinity perfection. ${ }^{[28 \mathrm{f}]}$ However, the major disadvantages of these approaches are the highly expensive facilities and the complex preparation processes that limit their practical applications.

In addition to CNT films, graphene films have also been fabricated via a simple vacuum filtration process. ${ }^{[28 \mathrm{~g}, 28 \mathrm{~h}]}$ Wallace et $a .^{[28 \mathrm{~g}]}$ first investigated the graphene film by the vacuum filtration process for use as an anode. It exhibited good mechanical properties (Young's modulus of $41.8 \mathrm{GPa}$ and tensile strength of 293.3 MPa), excellent electronic conductivity $\left(351 \mathrm{Scm}^{-1}\right)$ and distinguishable electrochemical properties compared with pure graphite and offering an energy density of 1162 $\mathrm{Whkg}^{-1}$. Although, it possesses good mechanical properties, its rate capability and specific capacities were unsatisfactory due to a restacking of the graphene sheets. Later reports also indicated that electrochemical performance of graphene papers as anode in LIB has a strong dependency on the thickness of the graphene paper with thinner papers having a better performance than thicker ones. ${ }^{[28 i]}$ The decline in performance with thicker papers can be attributed to the large aspect ratio and a dense restacking of graphene sheets. To avoid a restacking of the graphene, spacer or filler such as acetylene black NPs and CNTs (Figure 4a) ${ }^{[29]}$ were introduced in-between the nanosheets (NSs) of the graphene paper. For instance, a porous graphene $(\mathrm{PG})$ paper was synthesized via a freeze drying of a wet graphene oxide (GO) gel followed by a thermal reduction process. ${ }^{[29 \mathrm{c}]}$ The water content in the precursor GO was used to inhibit the restacking of graphene NSs. The macro porous structure of the fabricated PG paper can be tuned by adjusting the water content in the gel precursor. It can be used as a binder-free LIB anode that delivers a discharge capacity of $420 \mathrm{mAhg}^{-}$ 1 at $2000 \mathrm{mAg}^{-1}$ current density. The electrochemical performance is due to the $3 \mathrm{D}$ interconnected porous structure for rapid ion diffusion and fast charge transfer. The PG paper electrode is flexible and mechanically robust, making it an attractive electrode for flexible devices.

Free standing composite films. In free standing composite film electrodes, the $0 \mathrm{D}, 1 \mathrm{D}, 2 \mathrm{D}$ nanomaterials are homogenously mixed with CNT or graphene. ${ }^{[30]}$ Due to limited specific capacity of graphite $\left(372 \mathrm{mAhg}^{-1}\right)$ the carbon film-based pliable anodes have limited specific capacity. Instead $\mathrm{Sn}, \mathrm{Si}, \mathrm{Ge}$, transition metal oxides $\left(\mathrm{Co}_{3} \mathrm{O}_{4}, \mathrm{Fe}_{3} \mathrm{O}_{4}\right.$, etc. $)$ and layered transition metal dichalcogenide $\left(\mathrm{MoS}_{2}, \mathrm{SnS}_{2}\right.$ etc. $)$ based anodes with high specific capacities have been used. However, large volume expansions, structure degradation during charging/ discharging processes and the continual formation of the solid electrolyte interphase (SEI) are the major drawbacks for these high capacity materials. Porous CNT and graphene with large surface areas, high flexibility and lightweight are more suited as a substrate for these high

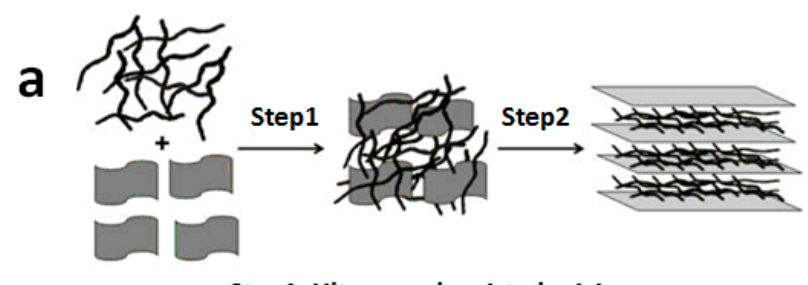

Step1: Ultrasound assisted mixing Step2: Vacuum assisted filtration

b

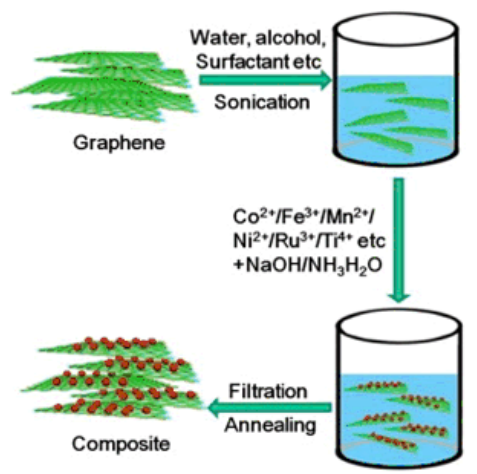

Figure 4. (a) Schematic diagram of fabrication of the CNT-GN papers by the vacuum-assisted filtration. Reproduced with permission. ${ }^{[29 b]}$ Copyright 2013, Elsevier B.V. (b) A general wet-chemistry strategy to fabricate graphene/metal oxide composites. Reproduced with permission. ${ }^{[30 \mathrm{~d}]}$ Copyright 2012, Elsevier B.V. 
capacity materials.

Vacuum filtration is the most common technique for fabricating CNT or graphene-based composites and used as BEs for a flexible LIB (Figure 4b). ${ }^{[30-d]}$ For instance, $\mathrm{Si} /$ Reduced graphene oxide (RGO) composite film was fabricated via simply vacuum filtration and subsequent thermal reduction method, in which Si nanoparticles (NPs) were encapsulated in RGO matrix and showed better electrochemical performance than its individual counterparts. ${ }^{[30 \mathrm{e}]} 1 \mathrm{D} / 2 \mathrm{D}$ hybrid structure using $\mathrm{Si}$ nanowires and graphene was also synthesized through vacuum filtration method followed with heat treatment. ${ }^{[30 f]}$ This paper like electrode exhibited many advantages; not only it provided a short diffusion path to the $\mathrm{Li}^{+}$ion and electrons, but also, prevented a volume change in the $\mathrm{Si}$. It is often used as a binder free anode in the LIB and showed a high reversible capacity (ca. $3350 \mathrm{mAhg}^{-1}$ of $\mathrm{Si}$ ), excellent stability and high rate performance. A similar method was also used to hybridize Ge NPs with SWCNT and paired with the $\mathrm{LiFePO}_{4}$ cathode to deliver an anode capacity of $800 \mathrm{mAhg}^{-1}$ at a discharge rate of $1 \mathrm{C}^{[30 \mathrm{~g}]}$ Although, the graphene and CNT matrix accommodate the volume expansion, but because of the direct contact with the electrolyte, a continual formation of the SEI film occurs and this reduces the battery performance.

To avoid the direct electrolyte contact with the active material a dual strategy has been used, in which the active material is coated with carbon and then, encapsulated with CNT or graphene. ${ }^{[30 \mathrm{~h}]}$ The Si nanowires were encapsulated with graphene $(\mathrm{G})$ sheaths, overcoated with RGO and used as a free standing anode (SiNW@G@RGO) for LIB. ${ }^{[30 i]}$ The graphene sheaths avoided the direct contact of Si with the electrolyte and the flexible RGO overcoat accommodated the volume expansion of embedded SiNW@G nanocables. The result is a SiNW@G@RGO anode that is capable of delivering a high specific capacity (1600 mAhg ${ }^{-1}$ at $2.1 \mathrm{Ag}^{-1}$ with an $80 \%$ capacity retention after 100 cycles) and a superior rate performance (500 mAhg ${ }^{-1}$ at $\left.8.4 \mathrm{Ag}^{-1}\right)$. In another approach, a flexible $\mathrm{Si} / \mathrm{SiO}_{2} / \mathrm{C}$ nanofiber electrode material was fabricated by electrospinning and heat treatment methods. ${ }^{[30 j]}$ To further increase the cycle life, an additional carbon nanocables was overcoated on $\mathrm{Si} / \mathrm{SiO}_{2} / \mathrm{C}$ by the CVD method. The carbon overcoating on the composite keeps the structural integrity intact and this increases the cycling performance. Lukman et al. ${ }^{[30 \mathrm{k}]}$ reported $\mathrm{SWCNT} / \mathrm{SnO}_{2}$ anode paper through the vacuum filtration of SWCNT/SnO 2 composite material. This composite material was first synthesized by the polyol method and then filtrated to form a paper with the CNTs forming a 3D nanoporous network. The anode paper with $\mathrm{SnO}_{2}$ of $34 \mathrm{wt} . \%$ exhibited a much better electrochemical performance than the pristine CNT paper. The SWCNT accommodated the volume expansion and at the same time, provided an efficient electrically conducting paths.

Additionally, flexible composite films of metal oxides such as $\mathrm{ZnO},{ }^{[301]} \mathrm{TiO}_{2},{ }^{[30 \mathrm{~m}]} \mathrm{Co}_{3} \mathrm{O}_{4},{ }^{[30 \mathrm{n}]} \mathrm{MnO}_{2},{ }^{[30 \mathrm{o}]} \mathrm{Fe}_{3} \mathrm{O}_{4},{ }^{[30 \mathrm{p}]}$ etc. with CNT/graphene have also been reported and used as bendable free standing anodes for LIBs. For example, holly $\mathrm{Fe}_{3} \mathrm{O}_{4} /$ graphene $\left(\mathrm{H}-\mathrm{Fe}_{3} \mathrm{O}_{4} / \mathrm{GS}\right)$ film was fabricated via vacuum filtration, followed by a thermal reduction process for use as a flexible free standing anode in the LIB. $^{[30 \mathrm{ql}]}$ It delivers a high specific capacity of $1555 \mathrm{mAhg}^{-}$ ${ }^{1}$ at a current density of $100 \mathrm{mAg}^{-1}$, and it showed excellent cyclic stability and rate performance. ${ }^{[30 \mathrm{q}]}$ This superior electrochemical performance was attributed to the 3D porous graphene network, which maintains good electrical contacts in $\mathrm{H}-\mathrm{Fe}_{3} \mathrm{O}_{4}$ with reduced diffusion paths for the electrons and $\mathrm{Li}^{+}$ions. In addition to the vacuum filtration approach, a uniform distribution of $\mathrm{Fe}_{2} \mathrm{O}_{3} \quad \mathrm{NPs}$ in $\mathrm{Fe}_{2} \mathrm{O}_{3}$ /SWCNT membrane was obtained by the CVD approach. ${ }^{[30 r]}$ The Fe/SWCNT membrane was fabricated by CVD technique and the paper-like film was obtained by oxidizing in air the $\mathrm{Fe}$ to $\mathrm{Fe}_{2} \mathrm{O}_{3}$. This paper-like film is highly flexible and used as a binder free and current collector free anode to yield a high reversible capacity (1243 $\mathrm{mAhg}^{-1}$ at $50 \mathrm{mAg}^{-1}$ ) and excellent cyclic stability. Just like the CNT or graphene, the expanded graphite (EG) is low cost and more importantly, to support the active materials for an improved electrochemical performance of the electrodes due to its high electrical and thermal conductivity, porous structure and mechanical flexibility. ${ }^{[31]}$ Zhao et al.${ }^{[32]}$ reported an EG-based paper for use as a free standing anode and to further increase the $\mathrm{Li}$ storage capacity, $\mathrm{Co}_{3} \mathrm{O}_{4}$ NPs were delicately anchored between the NSs of EG. After 50 cycles its reversible capacity was increased $19.2 \%$ and this can be attributed to 3 factors: spacer effect $\left(\mathrm{Co}_{3} \mathrm{O}_{4}\right)$, which facilitated the electrolyte diffusion, high mechanical strength and elasticity of EG buffered the volume expansion of the active material during the charging/discharging process, and the high conductivity of EG provided a fast transfer path for the electrons and ions.

To further enhance the battery performance, the synergistic effect of two metal oxides together with graphene has been investigated. A free standing flexible $\mathrm{Fe}_{2} \mathrm{O}_{3} @ \mathrm{SnO}_{2} / \mathrm{GS}$ film was fabricated by vacuum filtration of the spindle-like $\mathrm{Fe}_{2} \mathrm{O}_{3} @ \mathrm{SnO}_{2} \mathrm{NPs}$ and the GO mixed solution is subjected to a thermal reduction, to homogeneously dispersed the spindle-like $\mathrm{Fe}_{2} \mathrm{O}_{3} @ \mathrm{SnO}_{2}$ NPs in-between the graphene NSs. ${ }^{[33]}$ The result is a highly efficient structure for the diffusion of $\mathrm{Li}^{+}$ions and electrons, and it prevented the agglomeration of NPs. The binder free anode exhibited excellent electrochemical performance (1015 $\mathrm{mAhg}^{-1}$ even after 200 cycles) due largely to the synergistic effects between $\mathrm{Fe}_{2} \mathrm{O}_{3}$ and $\mathrm{SnO}_{2}$. Recently, a mixed metal oxide/oxidized graphene (OGP) composite was used as a flexible and binder free flexible negative electrode. ${ }^{[34]}$ The composite consisting of nickel manganese ferrite $\left(\mathrm{Ni}_{0.5} \mathrm{Mn}_{0.5} \mathrm{Fe}_{2} \mathrm{O}_{4}\right.$ (NMFO)) NPs grown on the OGP network was prepared by a new solvothermal approach. The flexible and binder free electrode was fabricated by depositing the OGP and NMFO/OGP on 
polypropylene film via the vacuum filtration technique. The obtained binder free NMFO/OGP coated polypropylene film and free-standing NMFO/OGP possesses a high mechanical flexibility and an improved electrochemical performance compared with pristine NMCFO NPs.

The composite films of CNT/graphene or CNF with layered metal dichalcogenides such as $\mathrm{MoS}_{2}, \mathrm{SnS}_{2}$ etc. have also been prepared by electrospinning ${ }^{[30 \mathrm{~s}]}$ and the vacuum filtration process ${ }^{[30,30 u]}$ for use as a free standing anode. In a typical example, a highly flexible porous carbon nanofiber (PCNF)@ $\mathrm{MoS}_{2}$ coaxial fiber membrane was prepared via a combination of electrospinning and solvothermal approach, in which the $2 \mathrm{D} \mathrm{MoS} 2 \mathrm{NSs}$ were homogenously coated over $1 \mathrm{D}$ porous $\mathrm{CNF}$ and resulting in a 3D open structure. ${ }^{[30 s]}$ The 3D open structure facilitates a fast charge transport, increases the surface active sites of $\mathrm{MoS}_{2}$ and prevents the agglomeration of $\mathrm{MoS}_{2}$ NPs during the charging/discharging process. It exhibited a high rate capability (475 mAhg ${ }^{-1}$ at $\left.1 \mathrm{Ag}^{-1}\right)$ and cycling stability. Taking advantage of the incorporated CNT in-between the graphene $\mathrm{NSs},{ }^{[29 b]}$ a flexible paper was fabricated, in which the $\mathrm{SnS}_{2}$ NSs were uniformly distributed and confined within the conductive graphene-CNT hybrid structure via a facile vacuum filtration and calcination approach. ${ }^{[30 t]}$ The aggregation and pulverization of $\mathrm{SnS}_{2} \mathrm{NSs}$ during a cycling process was effectively prevented by the confinement of $\mathrm{SnS}_{2} \mathrm{NSs}$ in the graphene-CNT frameworks. As a result, an improved electrochemical performance in terms of capacity and cycling stability was obtained.

\subsection{Compressible/Bendable Electrodes (CBEs) Based on 3D Bulk Materials}

Recently, 3D bulk carbon materials such as the graphene foam, CNT sponge and aerogels ${ }^{[35]}$ etc. have received considerable attention for constructing flexible LIBs as they are able to sustain high bending and compressive loads. The CNT and graphene building blocks of the 3D bulk material provide a large surface area, 3D interconnected porous structure with excellent bendability and compressibility and high electronic and ionic conductivity to facilitate fast movements of ions and electrons. ${ }^{[36]}$ However, pure carbon based electrodes have limited specific capacity $\left(372 \mathrm{mAhg}^{-1}\right)$ and it can be increased by a hybridization of the 3D bulk materials with high capacity active materials in an attempt to engineer target materials with multifunctionalties and improved properties for practical applications. ${ }^{[37]}$ Binder free 3D composite electrodes that use carbon cloth (CC) or textile as a template for depositing the active materials have been very popular. ${ }^{[38]}$ Liu et al. ${ }^{[38 a]}$ fabricated a $3 \mathrm{D} \mathrm{ZnCo}_{2} \mathrm{O}_{3}$ nanowire array/CC hierarchical composite using a simple hydrothermal process for use a free standing pliable anode with a high reversible capacity (1300-1400 $\mathrm{mAhg}^{-1}$ ) and excellent cyclic stability (1200 mAhg ${ }^{-1}$ after 160 cycles). A fully assembled battery using $\mathrm{ZnCo}_{2} \mathrm{O}_{3}$ nanowire array/CC as anode and $\mathrm{LiCoO}_{2} / \mathrm{Al}$ foil as cathode (Figure 5a) is shown to operate between a 2.2-3.7 V voltage window with a good capacity retention (of $1300 \mathrm{mAhg}^{-1}$ that is kept at $96 \%$ after 40 cycles). Further, after several hundreds of
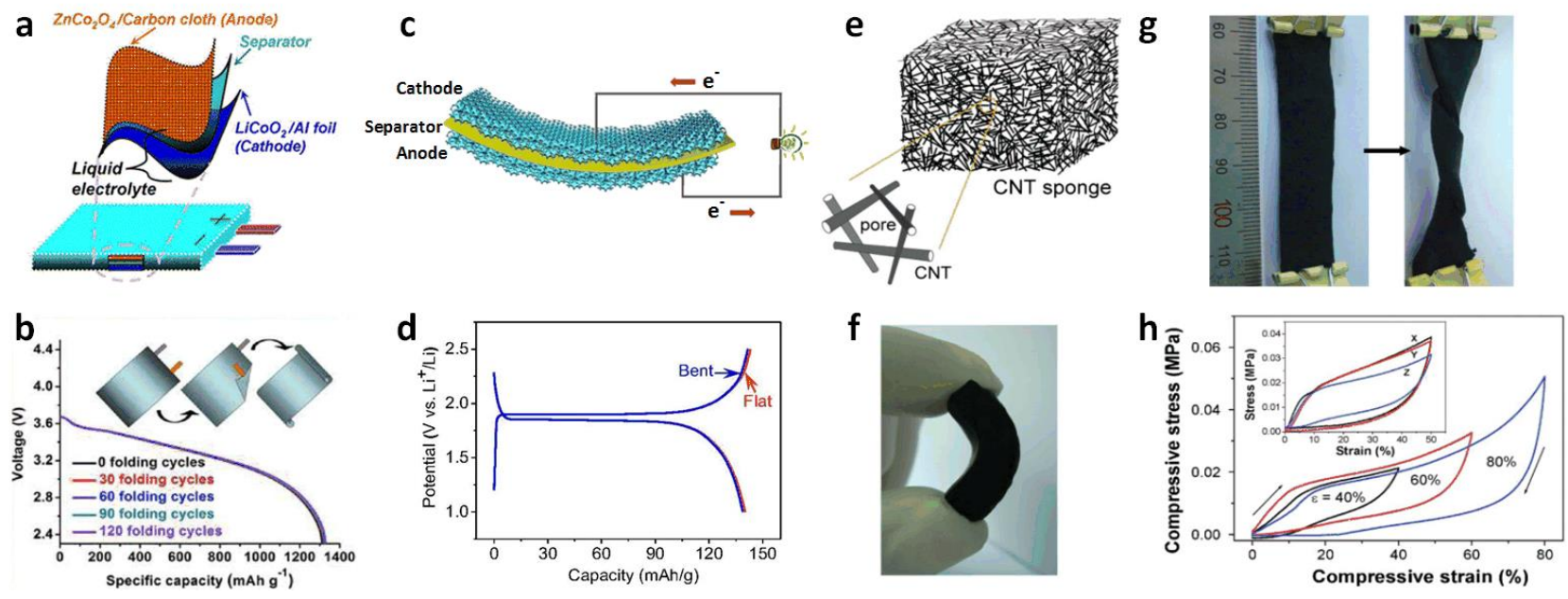

Figure 5. (a) Structure of the flexible Li-ion battery. (b) The voltage versus specific capacity profiles of full flexible

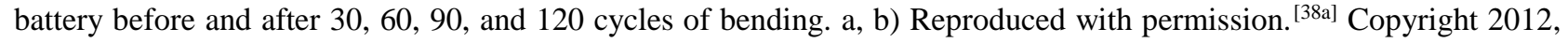
American Chemical Society. (c) Schematic of a flexible battery containing a cathode and an anode made from 3D interconnected GF. (d) Galvanostatic charging/discharging curves of the battery. c, d) Reproduced with permission. ${ }^{[40]}$ Copyright 2012, PNAS (e) Illustration of the sponge consisting of CNT piles (black lines) as the skeleton. (f) A CNT sponge bent to arch-shape. $(\mathrm{g})$ A $5.5 \times 1 \times 0.18 \mathrm{~cm}^{3}$ sponge twisted by three round turns at the ends without breaking. (h) Loading and unloading compressive stress-strain curves of several sponges at different set strains. e-h) Reproduced with permission. ${ }^{[35 b]}$ Copyright 2010, John Wiley\& Sons, Inc. 
cycles, it did not exhibit significant performance degradation as measured from the voltage profile, and mechanical and electrical robustness and stability (Figure 5b). A unique hierarchical 3D carbon structure was developed using vertically-aligned CNT (VACNT) arrays grown directly on $\mathrm{CC}$ by a plasma enhanced chemical vapor deposition (PECVD) technique and then, Sicoated. ${ }^{[38 i]}$ This VACNT-Si/CC hybrid structure was used as a pliable anode and it delivered a high areal capacity of $3.33 \mathrm{mAhcm}^{-2}$ at $0.2 \mathrm{mAcm}^{-2}$ current density. The enhanced performance can be attributed to the high electronic conductivity, high surface area and mechanically robust and structural stability of Si.

Besides CC or carbon textile, 3D graphene network (3DGN) grown by CVD has also been used as a template to deposit nanomaterials. ${ }^{[35 a, 39]}$ The 3DGN consists of interconnected graphene NSs and serves not only as a substrate for the active electrode material deposit, but it also, provides large surface area and preserves the graphene conductivity. Further, it is lightweight, highly flexible and good conductivity for flexible LIBs. Li et al. ${ }^{[40]}$ assembled a full battery using a 3D flexible graphene foam as a current collector and a graphene foam loaded with LTO NSs and LFP NPs, respectively, as an anode and a cathode (Figure 5c) with a total thickness of $<800 \mu \mathrm{m}$ and a specific energy density of $\sim 110 \mathrm{Whkg}^{-1}$. They showed that the flexible battery was able to power a red LED under continuous bending to $<5 \mathrm{~mm}$ radius of curvature without any appreciable structural degradation. The battery operated at a stable voltage of $1.9 \mathrm{~V}$ under bending with an initial discharge capacity of $\sim 143 \mathrm{mAhg}^{-1}$ at $98 \%$ coulombic efficiency with a rate of $0.2 \mathrm{C}$. Its capacity decreased only by $1 \%$ after being bended 20 times at a radius of $5 \mathrm{~mm}$ as a demonstration of the high mechanical stability and flexibility (Figure 5d).

Recently, CNT sponges with 3D macroscopic porosity $>99 \%$, interconnected CNT skeletons, high electrical conductivity $\left(\sim 200 \mathrm{Sm}^{-1}\right)$, low density $\left(5-10 \mathrm{mgcm}^{-3}\right)$, thermal stability, excellent mechanical flexibility and robustness have been reported for fabricating flexible and compressible 3D porous substrate (Figure 5e). ${ }^{[35 b]}$ The macroscopic structure and high electrical conductivity of the CNT sponge meant short diffusion paths for ions and high conductive pathways for electrons to enhance the rate performance. It exhibited good mechanical properties under bending (Figure 5f), no damage to its structural integrity after twisting up to 3 twists (Figure 5g) and recovered elastically under compression (Figure 5h). Also, due to its high surface area porosity a significant amount of active mass can be loaded onto the CNT sponge. With all these good characteristics, several compressible/ bendable electrochemical energy storage devices have been fabricated.$^{[41]}$ Lou et al. ${ }^{[41 \mathrm{~b}]}$ built a carbon coated, CNT/tin (oxide) spongy coaxial structure (CNT/Sn@C) via hydrolysis with a subsequent carbonization and annealing method for use as a free standing anode. The CNT sponge provided the mechanical flexibility, robustness and a conducting path for ions and electrons, while the carbon coating avoided a direct contact between the active material and the electrolyte. They reported a high reversible capacity of $847 \mathrm{mAhg}^{-1}$ at $100 \mathrm{mAg}^{-1}$ and a high cyclic stability (818 mAhg ${ }^{-1}$ after 100 cycles) with a high rate gravimetric and areal capacity.

Our group has also fabricated pliable and free standing electrodes synthesized from CNT sponge embedded with $\mathrm{MoS}_{2}$ nanoparticles $\left(\mathrm{CNT} @ \mathrm{MoS}_{2}\right)$. The CNT@MoS 2 electrodes are fabricated via a 2 -step synthesis process that involves a novel low temperature thiourea thermal technique. The CNT sponge was impregnated with the $\mathrm{MoS}_{2}$ precursor solution to obtain $\mathrm{MoS}_{2}$ moieties on the CNT substrate. Subsequently, the dried CNT@ $\mathrm{MoS}_{2}$ was immersed in a melted thiourea solution and transferred to a Teflon-lined stainless steel autoclave for reaction at $120^{\circ} \mathrm{C}$ for $12 \mathrm{~h}$. The obtained product was immersed in $\mathrm{CS}_{2}$ to eliminate elemental $\mathrm{S}$ components and air dried followed by heat treatment at $800{ }^{\circ} \mathrm{C}$ at $6 \mathrm{~h}$ to improve the crystallinity. The morphological characterization clearly suggested that the CNTs retain their original structure with an average diameter of $20 \mathrm{~nm}$ (Figure 6a). The TEM images further revealed the uniform coating of $\mathrm{MoS}_{2}$ on CNT surface (Figure 6b).The as-fabricated CNT@ $\mathrm{MoS}_{2}$ sponge exhibited excellent structural stability under bending (Figure 6c) due to flexible nature of CNT sponge $^{[35 b]}$ as a support material.

The electrochemical properties of the freestanding anode made from $\mathrm{MoS}_{2} @ \mathrm{CNT}$ sponge for LIBs was evaluated by cyclic voltammetry $(\mathrm{CV})$ and galvanostatic discharge/charge cycling. The first three cyclic voltammetry (CV) curves of $\mathrm{MoS}_{2} @ \mathrm{CNT}$ sponge measured under a scan rate of $0.3 \mathrm{mVs}^{-1}$ in the voltage window of 0.01 3 V, are depicted in Figure 6d. In the initial cathodic sweep, two peaks located at 0.67 and 0.12 $\mathrm{V}$ emerged. The first peak can be assigned to the lithiumion intercalation during the $\mathrm{MoS}_{2}$ process to form $\mathrm{Li}_{x} \mathrm{MoS}_{2}$. The second peak with a higher intensity corresponds to the reduction of $\mathrm{Li}_{x} \mathrm{MoS}_{2}$ into metallic Mo NPs and $\mathrm{Li}_{2} \mathrm{~S}$ matrix via a conversion reaction, and then the formation of a gel-like polymeric layer resulting from electrochemically driven electrolyte degradation. In the reverse anodic sweep, only one prominent peak at $2.45 \mathrm{~V}$ is seen and this can be attributed to the extraction of $\mathrm{Li}_{2} \mathrm{~S}$ and oxidation of Mo to $\mathrm{MoS}_{2}$. During subsequent cycles, the original cathodic peak at 0.12 disappears while two new peaks at 1.77 and $0.85 \mathrm{~V}$ are repeatedly observed, which is consistent with the literature. ${ }^{[42]}$ The peaks are also identified with plateaus in the first three cycles of discharge/charge curves (Figure 6e). Also, an anodic peak at $1.71 \mathrm{~V}$ appears due to the restacking of $\mathrm{MoS}_{2}$ with an enlarged interlayer distance $(0.64 \mathrm{~nm})$ which leads to much larger lithium-ion interaction with subsequent cycling.

It exhibited a first charge and discharge capacity of $623.7 \mathrm{mAhg}^{-1}$ and $643.4 \mathrm{mAhg}^{-1}$ respectively, at current 
(a)

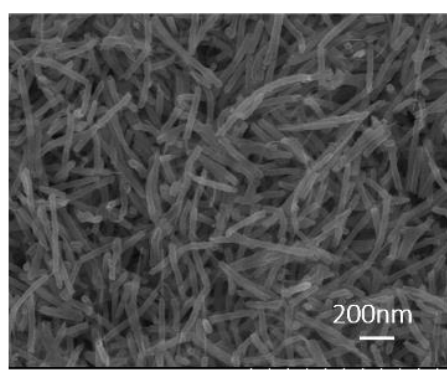

(d)

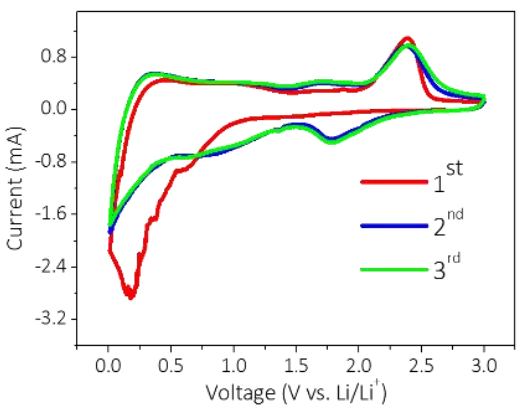

(b)

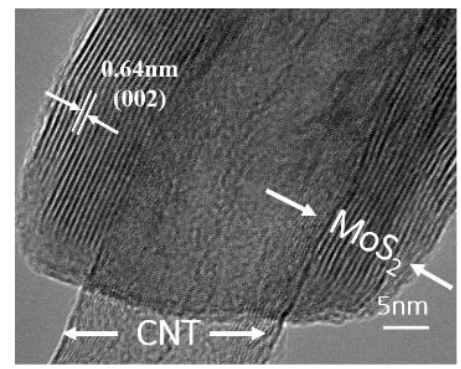

(e)

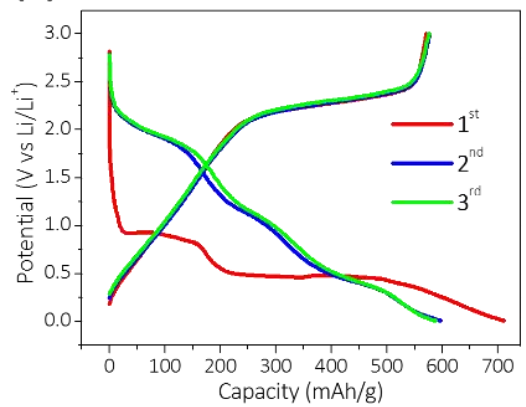

(c)

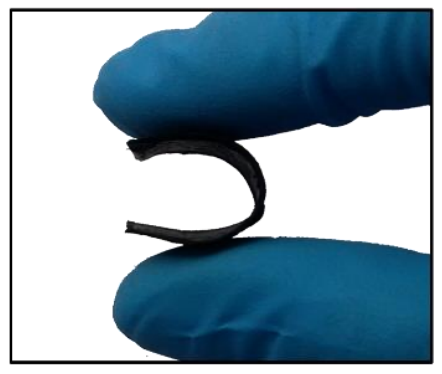

(f)

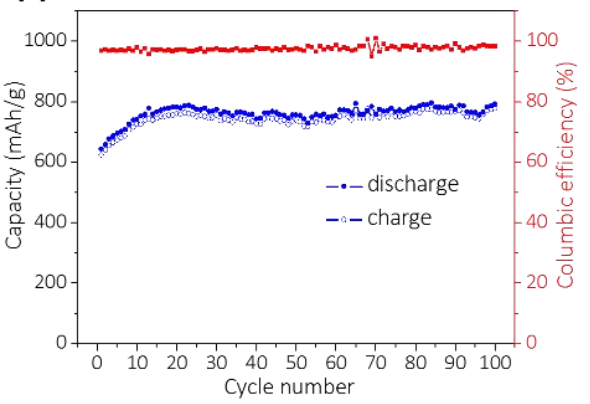

Figure 6. (a, b) SEM and TEM micrographs of CNT @ $\mathrm{MoS}_{2}$ revealing its core-shell structure. (c) A sponge being bended $180^{\circ}$ as a demonstration of its flexibility. (d-f) Electrochemical performances of CNT@ $\mathrm{MoS}_{2}$ sponge. (d) Cyclic voltammetry (CV). (e) $1^{\text {st }}, 2^{\text {nd }}$ and $3^{\text {rd }}$ charge/discharge curves. (f) Galvanostatic discharge/charge cycling at $100 \mathrm{mAg}^{-1}$.

density of $100 \mathrm{mAg}^{-1}$. The discharged capacity is further enhanced with the increasing cycles (Figure 6f). As shown, a $23.16 \%$ discharge capacity is increased from its initial value after 100 cycles. This is attributed to a greater exposed surface area of $\mathrm{MoS}_{2}$ and the increased interlayer distance (due to its layered structure nature during charging/discharging). The high flexibility and compressibility, good mechanical properties and high electrochemistry performance indicate that a CNT@ $\mathrm{MoS}_{2}$ sponge could be used to power flexible devices.

\subsection{Fiber-Based Stretchable Electrodes (FSEs)}

The electrode structure along with the design and active materials are important factors for assessing the performance of flexible energy storage devices. The 2D paper and bulky based-electrode batteries can be bent, twisted or compressed and have been applied in many paper electronics. ${ }^{[18 b]}$ However, to meet the demands of wearable and stretchable electronic products, versatility, durability and robustness are additional requirements that drive the development of power sources of these devices so that they are able to perform well under enhanced bending, twisting, and even stretching loads with a greater shape versatility and an omni-directional flexibility. ${ }^{[43]}$ The commercial polymer fibers are flexible and lightweight, but they have low conductivity and may even be insulating. They can be made conductive by a CVD coating with a conductive layer and this creates a hurdle for them to be produced on a large commercial scale. Carbon based fibers are promising and have been used in many wearable electronic products, ${ }^{[43]}$ solar cell, ${ }^{[44]}$ sensor, ${ }^{[45]}$ and currently, the fiber shaped $\mathrm{LIBs}^{[14]}$ are receiving considerable attention from the wearable and flexible device manufacturers.

Several flexible CNT fiber based electrodes have been investigated as a half-cell using the $\mathrm{Li}$ wire/foil as a counter electrode. ${ }^{[46]}$ The first CNT based composite fiber was developed by incorporating $\mathrm{MnO}_{2}$ NPs onto the surface of MWCNT by an electrochemical deposition in an aqueous solution with the weight percentage of the NPs controlled by a cycle number in the electrochemical deposition process. This composite fiber is highly flexible and did not exhibit any structural degradation or loss of electrical conductivity during deformations. It was investigated as a positive electrode in micro LIB with the $\mathrm{Li}$ wire as a negative electrode, and the whole electrode system achieved a specific capacity of $218.32 \mathrm{mAhg}^{-1}$ at $5 \times 10^{-4}$ $\mathrm{mA} .{ }^{[46 a]}$ In order to further increase the electrochemical performance, a high specific capacity active material (amorphous $\mathrm{Si}(\alpha-\mathrm{Si})$ ) was deposited onto the surface of the CNT yarn by a CVD technique and the thickness of deposited Si was controlled by the deposition time. The CNT@ $\alpha$-Si yarn possess a higher electrical conductivity and showed good flexibility and mechanically strength that is as high as the widely used conventional yarn in the textile industry. Further, its Li storage capacity was 5 times higher than with graphite. In addition, $\mathrm{Si}$ was also deposited onto 
aligned and continuous MWCNT sheets by an electron beam evaporation process. ${ }^{[46 \mathrm{~b}]}$ This MWCNT/Si sheet was then scrolled to form a fiber shape electrode with a coreshell structure. The half-cell was manufactured using this composite fiber as a working electrode and the $\mathrm{Li}$ wire as both the reference and counter electrodes (Figure 7a). Although a FSE exhibits a high specific capacity, its cyclic stability still needs improvement. In all the above cases, the
Li wire electrode is not as flexible as a fiber and this affects the full flexibility of the devices in many applications.

Current FSEs can be conveniently divided into 2 types based on their structural arrangement: helical coaxial structure and parallel structure. In 2012, Kim et al. ${ }^{[47]}$ fabricated a cable type flexible full battery with a helical coaxial structure (Figure 7b). It has an omni-directional flexibility that can be bent into any shape so that it can be
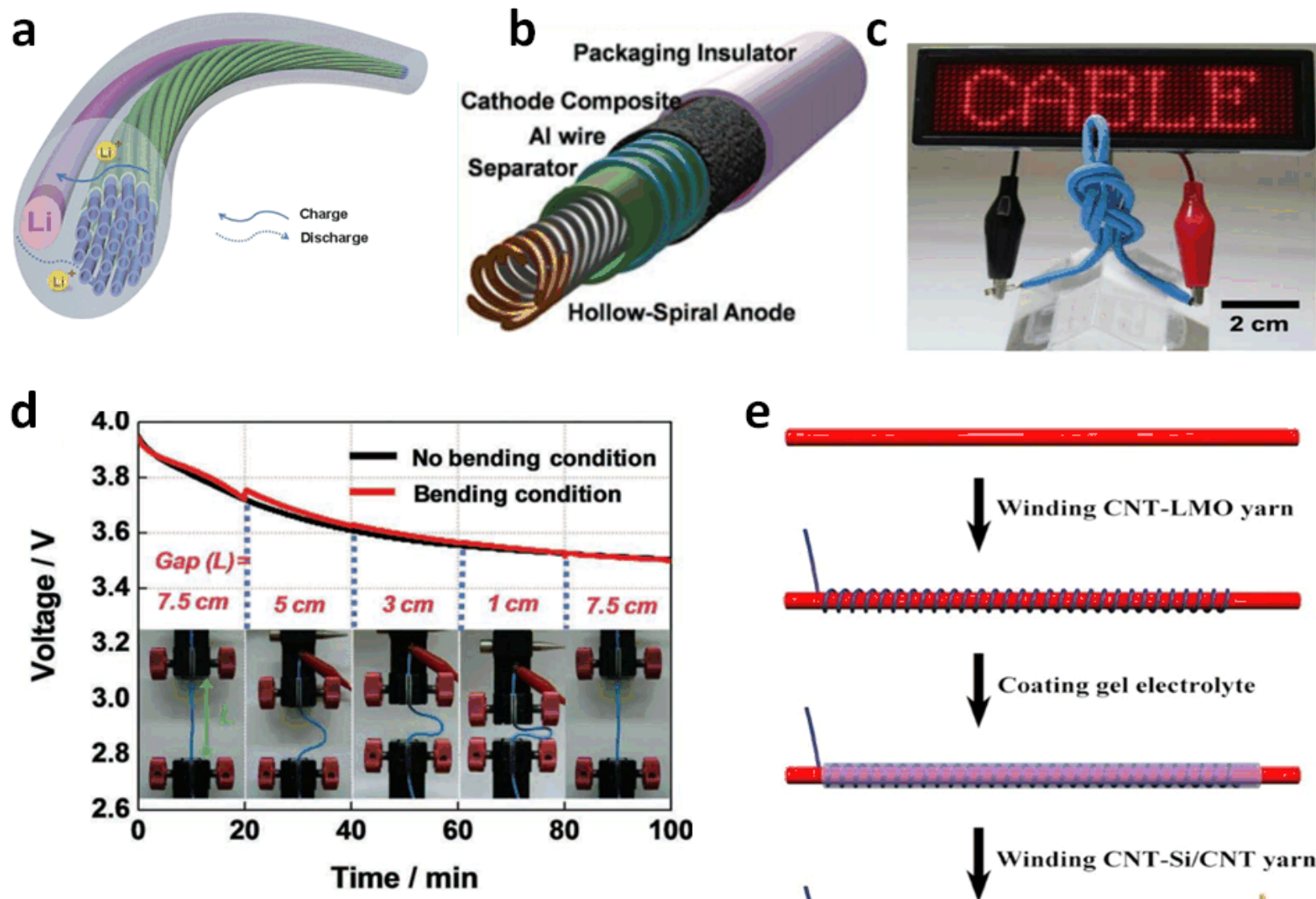

e

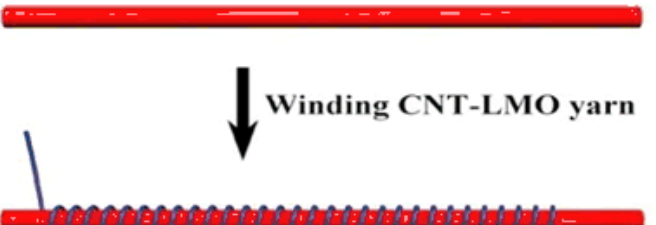

f
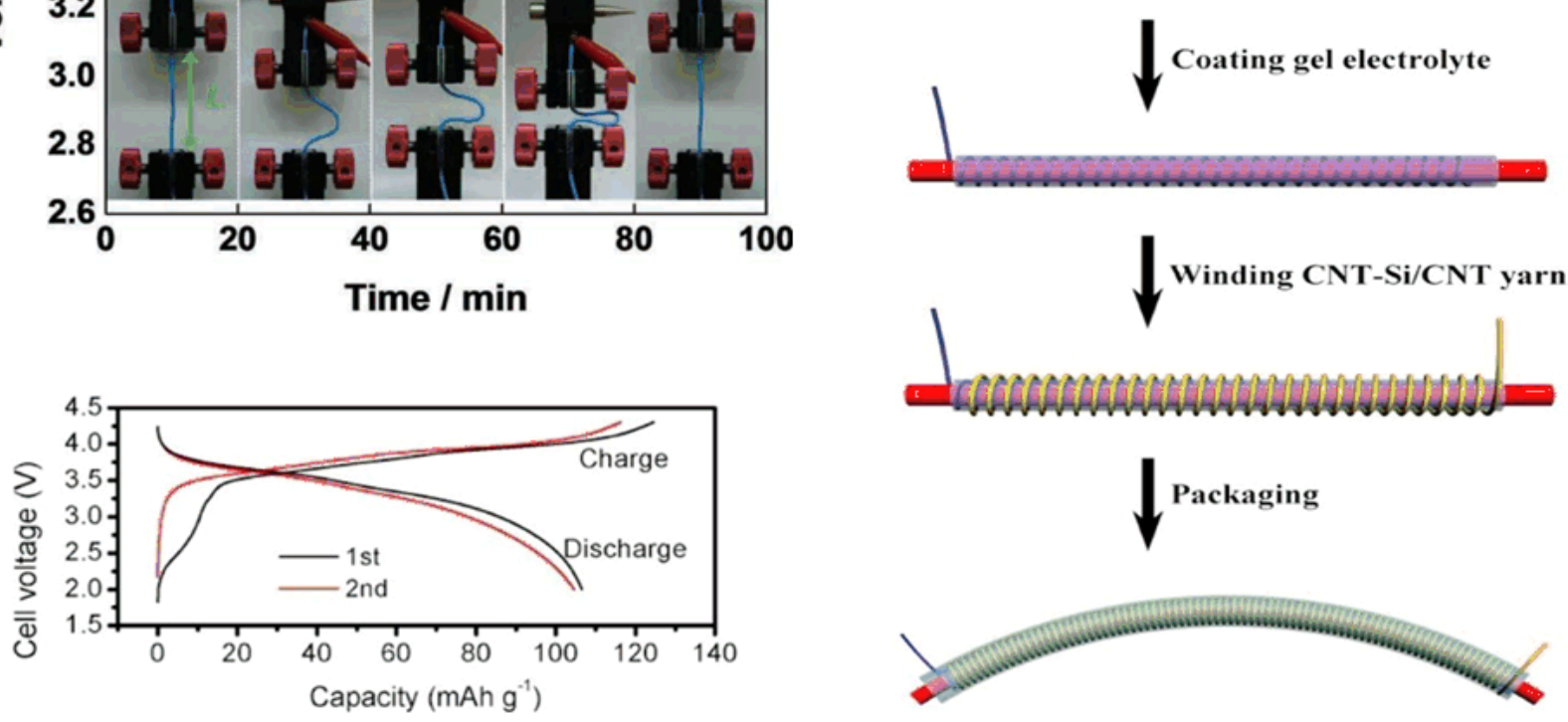

Figure 7.(a) Schematic illustration of a half lithium-ion battery. Reproduced with permission. ${ }^{[46 b]}$ Copyright 2014 , John Wiley \& Sons, Inc. (b) Schematic illustration of the cable battery with hollow-helix anode having multipl helix structure. (c) Photographs of a highly flexible cable battery under bending and twisting. (d) Discharge characteristics with variations in bending strain every 20 min. b-d) Reproduced with permission. ${ }^{[47]}$ Copyright 2012, John Wiley \& Sons, Inc. (e) Schematic illustration for the fabrication of the coaxial fiber full LIB. Red, blue, and yellow colors correspond to cotton fiber, CNT-LMO composite yarn, and CNT-Si/CNT composite yarn, respectively. Voltage profiles of the fiber-shaped LIB with $1 \mathrm{C}$ between 2.0 and 4.3 V. e, f) Reproduced with permission. ${ }^{[48]}$ Copyright 2014 , American Chemical Society. 
worn on any part of human body (Figure 7c). The battery exhibited a negligible variation in the discharge properties as the bending strain is increased (Figure 7d). However, it suffers from a low specific capacity $\left(1 \mathrm{mAhg}^{-1}\right)$ for the first charge/discharge cycle. Moreover, it is relatively large with a diameter in the millimeter range and this implies that the battery is unable to adequately meet the requirement with respect to the weavability. The weight and mass of battery can be further reduced by eliminating the metallic current collectors. Weng et al. ${ }^{[48]}$ fabricated a coaxial fiber LIB by sequentially winding an aligned CNT hybrid yarn anode $(\mathrm{CNT} / \mathrm{Si})$ and cathode $(\mathrm{CNT} / \mathrm{LMO})$ onto cotton fibers and separated by an electrolyte gel to avoid direct anode-cathode contact. The assembly is then covered by a protective layer of a shrinkable tube (Figure 7e). It exhibited initial specific capacity of $106.5 \mathrm{mAhg}^{-1}$ with an average voltage of $3.4 \mathrm{~V}$ (Figure 7f). Although, the device generated a better electrochemical performance, the full specific capacity of Si was still low. Further, the Si layer could be easily broken and this greatly decreases the stability of LIB.

Fiber shaped batteries generally work well under bending, twisting and tying deformations, but they don't seem to work so well under a stretching deformation. Two new designs have been created in an attempt to achieve better stretchability: substrate supported and substrate free LIBs. Employing a parallel structure, Ren et al. ${ }^{[49]}$ fabricated a flexible fiber LIB, in which the CNT/LTO yarn is made into the anode and the CNT/LMO yarn the cathode, and they are separated by a poly (vinylidene fluoride) membrane (Figure 8a). It is able to deform into various configurations with no apparent damage or performance loss. The LIB was made stretchable by winding the cathode fiber and anode fiber onto an elastic substrate with an additional coating of the gel electrolyte (Figure 8b). This battery demonstrated high stretchability up to a strain of $100 \%$ and maintained a $84 \%$ capacity after stretching for 200 cycles (Figure 8c). Other publications have reported on the stretchability issue using substrates such as silicon elastomers or elastomeric polymer to achieve the stretch. ${ }^{[49-50]}$ However, this approach has its share of problems; by using a substrate, it increases the volume and weight of the battery and decreases its energy density, which in turn, limits its practical application usage. Additionally, the low operating temperature and poor mechanical properties of polymeric substrate make them unstable.

Recently, a free standing LIB was fabricated using flexible hybrid spring as electrodes (Figure 8d) ${ }^{[51]}$ It was lightweight with a linear density of $10^{-2}-10^{-1} \mathrm{mgcm}^{-1}$, is flexible, stretchable and its electrochemical performance were maintained during both bending and stretching deformations. It can be easily stretched and released at strain of 50\% and 100\% (Figure 8e). The full battery has LMO/CNT and LTO/CNT as positive and negative electrodes, respectively and showed a specific capacity of
$92.4 \mathrm{mAhg}^{-1}$ with a good cyclic stability $(92.1 \%$ retained after 100 cycles). Further, as an indication of the high flexibility the capacity remained at $85 \%$ at the $100 \%$ strain, and less than $1 \%$ variation in capacity at the $50 \%$ strain after stretching continuously for 300 cycles (Figure 8f). These full fiber-shaped batteries appear to somewhat fulfill the needs of the wearer electronics by the textile industry as the flexible batteries can be woven into many flexible shapes and sizes of clothing to be worn on the human body. ${ }^{[48-49,50 b]}$ This flexible battery for clothing is still evolving and has become a future research direction for developing more efficient and better wearer LIBs.

\section{Electrode Materials for Supercapacitors}

Electrochemical capacitor (EC) or supercapacitor (SC) is a new class of capacitors that are capable delivering high power and energy densities, without the undesired safety concerns. ${ }^{[52]}$ The promising cycling life and electrochemical performance of SCs allows this type of energy storage devices to be attractive for charging future wearable electronics. ${ }^{[53]}$ However, the commercially available technology suffers from rigidity and heavy weight, therefore, it is desired to introduce lightweight, flexible, high performance all-solid-state SCs that can beg Sun easily integrated with wearable electronics. These flexible SCs can be used to power wearable electronics ${ }^{[54]}$ or miniature implantable biomedical devices. ${ }^{[55]}$

SCs utilize 2 main types of mechanism to store electrical energy and they are electrical double layer capacitance (EDLC) and pseudocapacitance (Figure 9). ${ }^{[56]}$ The energy in an EDLC is stored electrostatically in an electric field, leading to extremely short discharge times. Representative EDLC materials include activated carbon (AC), graphene (G), and CNT. ${ }^{[57]}$ Pseudocapacitance is the charge storage behavior achieved from the reversible Faradaic redox reactions on the electrode/electrolyte interface, yielding extremely high energy density, but suffer from long charging times. ${ }^{[58]}$ Popular pseudocapacitive materials for energy storage include conductive polymers such as polyaniline (PAni), polypyrrole (PPy), and transition metal oxides, such as ruthenium oxide $\left(\mathrm{RuO}_{2}\right)$, and manganese oxide $\left(\mathrm{MnO}_{2}\right) \cdot{ }^{[59]}$ Research has been underway on several fronts in order to improve the flexibility and mechanical properties of SC electrodes, while creating a hybrid system that takes advantage from both EDLC and pseudocapacitance storage mechanisms. ${ }^{[8,60]}$ In the following section, we highlight the different pliable electrode materials based on hydrogels (3D free standing), paper based (2D free standing) and flexible substrate supported and also discuss the future challenges for flexible SCs.

\subsection{Hydrogel Electrodes}

SC performance improves dramatically from the increase 


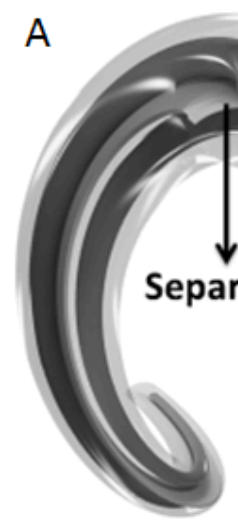

B
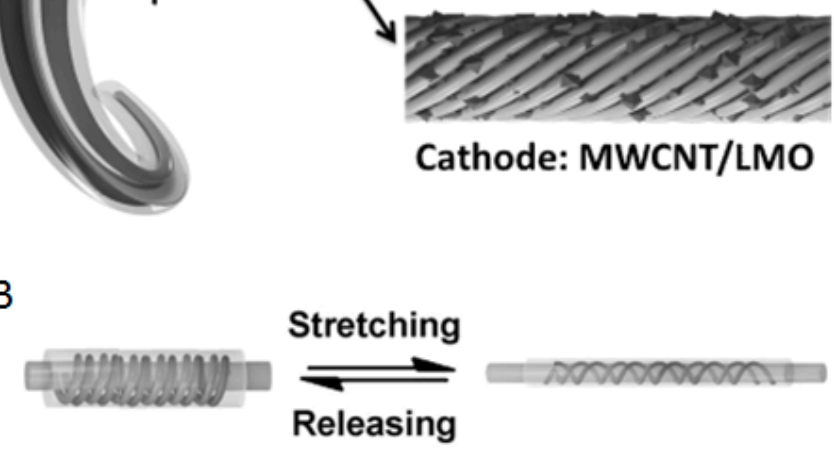

Cathode: MWCNT/LMO

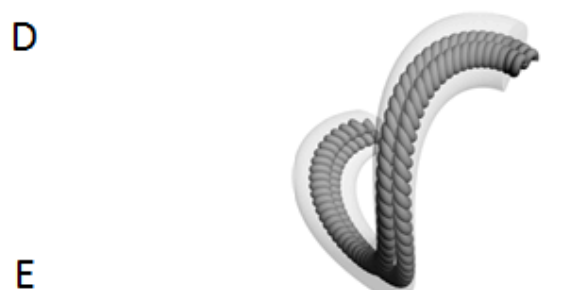

Anode: MWCNT/LTO

E
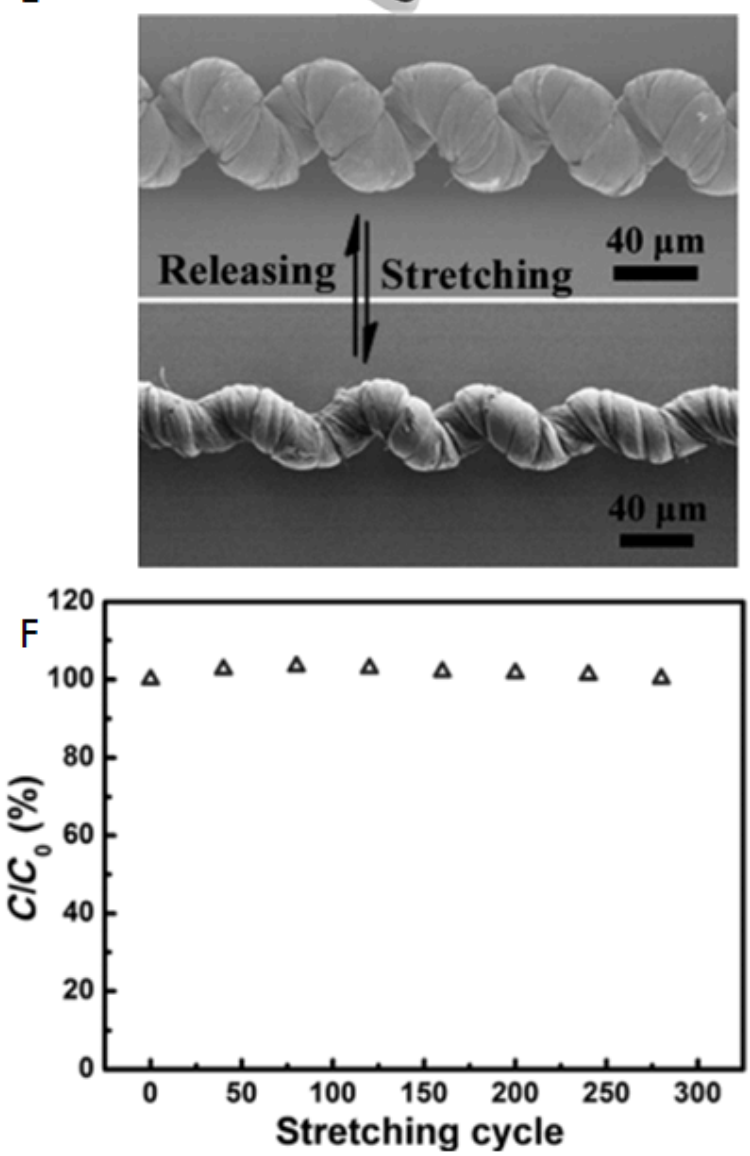

Figure 8. (a) Structure of the flexible wire-shaped LIB with parallel structure. (b) Stretching and releasing process. (c) Evaluation of the specific capacity with stretching cycles. $\mathrm{C}_{0}$ and $\mathrm{C}$ correspond to the specific capacities before and after stretch. a-c) Reproduced with permission. ${ }^{[49]}$ Copyright 2014, John Wiley \& Sons, Inc. (d) Structure of stretchable battery. (e) SEM images of a fiber 50\% and 100\% strains. (f) Dependence of specific capacitance with stretch cycles. df) Reproduced with permission. ${ }^{[51]}$ Copyright 2014, John Wiley \& Sons, Inc.

in electrode surface areas as a result of promoted EDLC, as well as improved Faradaic REDOX interaction of the electroactive species. A number of studies have utilized high-surface-area particles such as graphene or MWCNT to create electrodes for SCs, however, these typically required binder materials. ${ }^{[61]}$ The binder material does not actively participate in the charge storage process, and therefore would negatively impact the overall specific capacitance of the SC. Therefore it became attractive to construct 3D networked structures without the additional binder or inactive substrate materials.

Hydrogel represents a class of polymeric materials that contain hydrophilic 3D structural networks that allows the absorption of water molecules. ${ }^{[62]}$ Some of the benefits that hydrogel for pliable SC electrodes offer are the high flexibility and surface area that allows the ions electrolyte solution to fully interact with the active material within the 3D network. Free-standing hydrogels are typically fabricated through cross-linking the polymeric network chains. ${ }^{[62]}$ Researchers utilized both EDLC and pseudocapacitive materials in constructing hydrogel electrodes for flexible SC applications and have reported good electrochemical behaviors. ${ }^{[63]}$

Hydrogel electrodes are typically prepared via a simple chemical method and can be manufactured into very thin film electrodes that still retains a large ion-accessible 


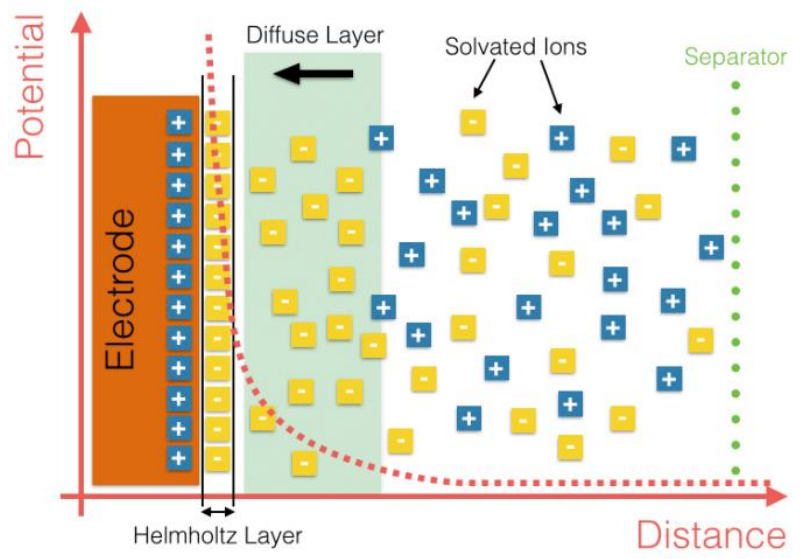

Electrochemical Double Layer (EDLC)

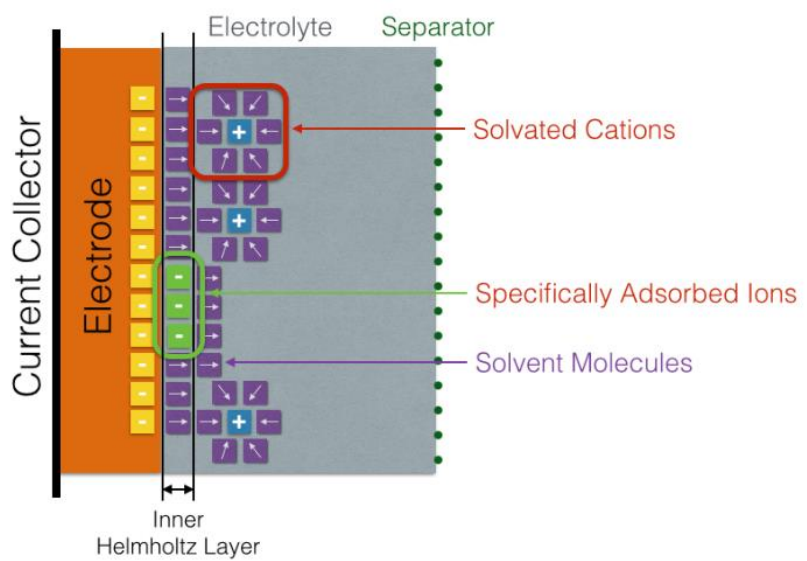

Pseudocapacitance

Figure 9. Charge storage mechanisms for SCs. EDLCs store energy electrostatically with the creation of Helmholtz layer at the electrode electrolyte interface, while pseudocapacitance achieves charge storage with the fast and reversible REDOX reactions at the electrode surface.

surface area. ${ }^{[63 b, 64]}$ The main advantages of such electrodes are the high electrical conductivity, the desirable mechanical properties, as well as the formation of hierarchical micro-porous 3D network structures. ${ }^{[63 c]}$ The flexibility can also be maintained through the integration with aqueous electrolyte systems, as the micro pores are allowed to fully swell and interact with the electrolytic ions $^{[65]}$. The high areal gravimetric specific capacitance values are obtained from the formation of hierarchical 3D structures that prevent graphene from restacking and expose the active surfaces for electrolytic interaction. The free movement of the electrolytic ion inside the electrode's porous networks further enhances the rate and cycling capabilities. However, hydrogel electrodes tend to suffer from aqueous electrolyte leakages if the cell is not packaged properly, leading to reduced cycle life. ${ }^{[64]}$ Scaling production that ensures the repeatability of hydrogel-based electrodes is also a challenge looking forward.

Early works by Ghosh et al. ${ }^{[65]}$ demonstrated the possibilities of extending the use of conductive polymer hydrogels to the field of energy storage. Shi et al. ${ }^{\text {63a] }}$ constructed SCs with PPy based hydrogel electrodes. These PPy hydrogels had an internal structure that is similar to foams, that forms a network of interconnected PPy microspheres. Flexibility was ensured via the use of $\mathrm{CC}$ as current collectors. The cyclic voltammetry showed a high specific capacitance range in between 300-400 F/g at a current density of $0.2 \mathrm{~A} / \mathrm{g}$. ${ }^{[63 \mathrm{a}]}$

With purely EDLC materials, Xu et al. ${ }^{[63 \mathrm{~b}, 64,66]}$ has successfully fabricated graphene hydrogel based pliable electrodes that can be applied as electrode for SCs. The graphene hydrogel was made through a simple one-step hydrothermal process at $180{ }^{\circ} \mathrm{C}^{[66]}$ with process details in Figure 10a, b. The polymer electrolyte system was casted on the graphene hydrogel electrode, and were pressed together with a similar counterpart to form the SC cell. The SEM images (Figure 10c-f) demonstrated the highly porous 3D structure of the SC hydrogel electrode that allowed efficient charge storage capabilities. As shown in Figure 10g, the flexible SCs with $120 \mu \mathrm{m}$ thick graphene hydrogel thin film electrodes achieved a high specific capacitance of $186 \mathrm{~F} / \mathrm{g}$, an aerial specific capacitance of $372 \mathrm{mF} / \mathrm{cm}^{2}$, and excellent cycling stability, while maintaining ideal mechanical flexibility. ${ }^{[64]}$ The ideal cycling behavior was also demonstrated in Figure $\mathbf{1 0 h}$ showing that nearly $100 \%$ of the capacitance was retained even after 10000 cycles. Xu et al. ${ }^{[63 b, 64,66]}$ constructed a porous hydrogel network that utilized the high specific surface area 3D structures to achieve an increased energy density compared to other types of electrodes (Figure 10i).

Researchers have employed various strategies in improving the hydrogel performance since then. Chen et $a l .{ }^{[67]}$ reported a nitrogen-doped graphene hydrogel structure that obtained a high power density of $205.0 \mathrm{~kW} / \mathrm{kg}$ even at very high charge/discharge rate of $185.0 \mathrm{~A} / \mathrm{g}$, while retaining 95.2\% capacitance after 4000 cycles. Nickel foams were also used as a substrate material for graphene hydrogels in order to improve the ionic access to the graphene hydrogel for improved EDLC performance. ${ }^{[68]}$ However, the rigidity of the metallic foams restricted the electrode's desired flexibility.

Hybrid hydrogels became an attractive option in the recent years as the combination of EDLC and pseudocapacitive materials proved to be an optimal synthesis strategy in creating high performance SCs. Hao et al. ${ }^{[63 \mathrm{~d}]}$ constructed a hybrid hydrogel system with PAni, polyacrylamide, and $\alpha$-cyclodextrin, leading to an increase in the specific capacitance at much lower active material loadings. Chen et al. ${ }^{[69]}$ created a novel graphene-nickel hydroxide $\left(\mathrm{Ni}(\mathrm{OH})_{2}\right)$ hybrid hydrogel that contains 

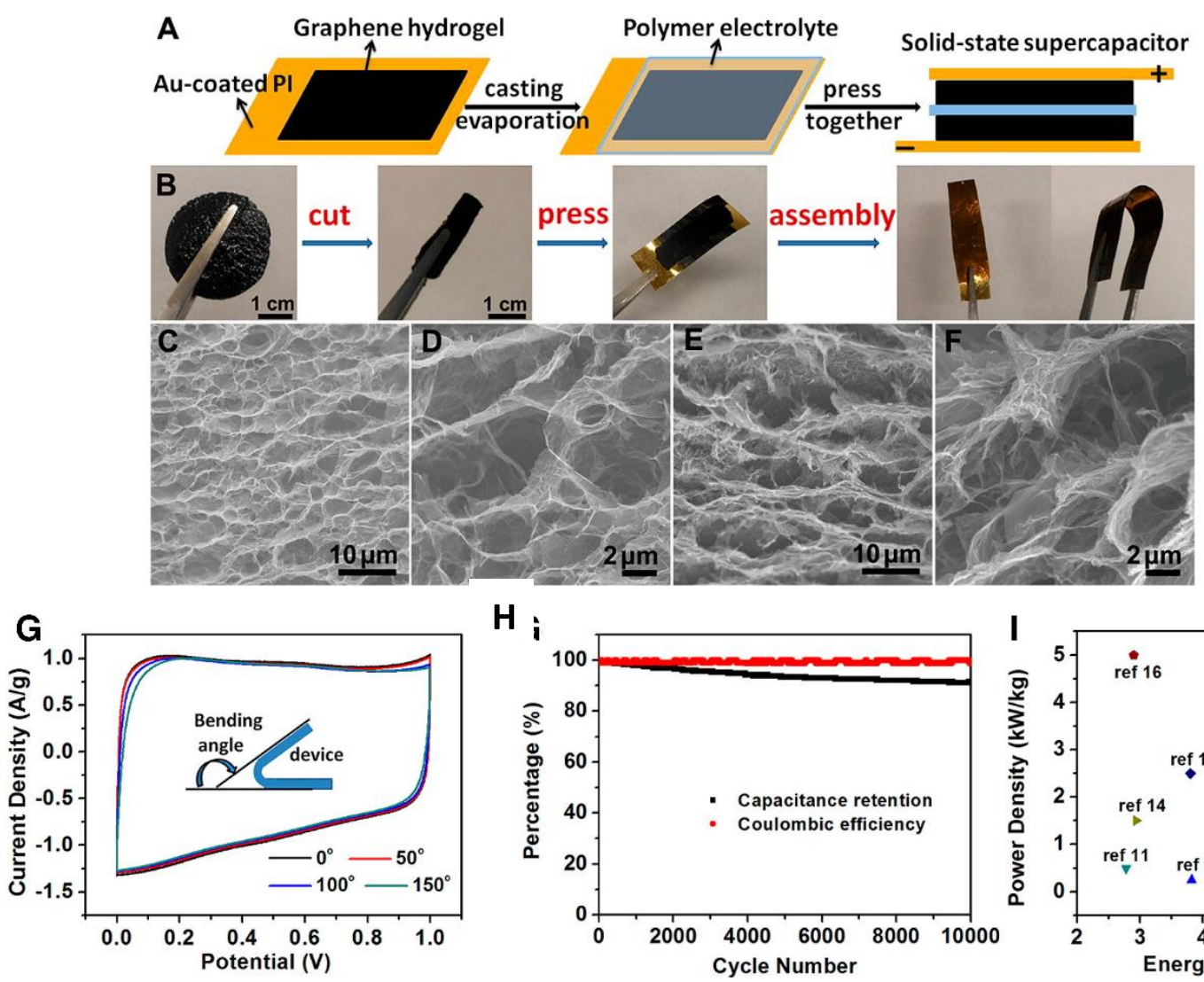

$\mathrm{H}$;
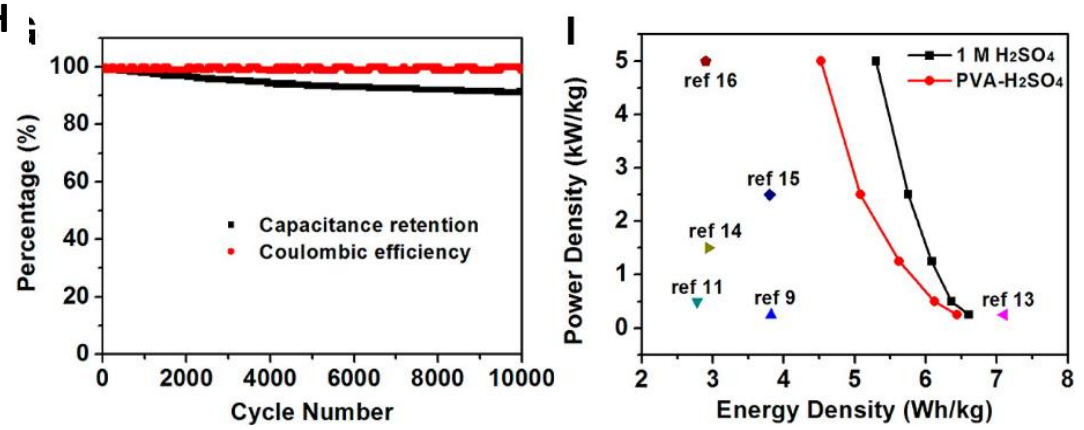

Figure 10. (a, b) The procedure for assembly of the SC cell with the flexible graphene hydrogel electrode materials made through the one-step hydrothermal process. (c-f) The scanning electron micrographs showing the highly porous 3D network morphology obtained in the flexible graphene hydrogel. (g) Ideal capacitive behavior at various bending angles of the electrode at $10 \mathrm{mV} / \mathrm{s}$ scanning rate. (h) Good cycling capabilities after 10,000 cycles, and high Coulombic efficiency with $10 \mathrm{~A} / \mathrm{g}$ charging/discharging current. (i) High power and energy densities in comparison with other existing SC devices. Reproduced with permission. ${ }^{[64]}$ Copyright 2013, American Chemical Society.

nanostructured pores that facilitates the ion transfer within the electrode's networked structure, thus leading to improved electrode kinetics.

\subsection{Paper-based Electrodes}

Paper products have good flexibility and have been made mechanically stronger and more resistant to wear and tear in the recent years. Researchers began to mimic the mechanical nature of paper, and started to experiment with paper-like electrodes made of active charge storage materials. To ensure flexibility, many researchers began integrating high-surface-area NPs such as VACNT or graphene particles with paper products in order to create SC electrodes with exceptionally high surface areas, and ultimately charge storage performances. ${ }^{[18 b, 19 a, 70]}$ Unlike the typically paper products, the wear and tear issues in paper-based EDLC electrodes can be effectively addressed with the superior mechanical properties of CNT or graphene. In order to further improve the electrode/ electrolyte interface, simple strategy was used to fabricate crumpled graphene paper SC electrodes that showed exceptionally high uniaxial strain at $\sim 300 \%$, while demonstrating a reasonable specific capacitance at 196 F/g. ${ }^{[71]}$ As shown in Figure 11, the graphene paper would be pre-stretching the graphene paper and let it relax biaxially to create the folding patterns to enhance the electrode performance. ${ }^{[71]}$

Other groups have looked at other ways of creating this type of lightweight, paper like hybrid structures for SC electrodes using EDLC materials, such as graphene or partially reduced graphene oxide, in combination with pseudocapacitive materials, such as PAni or vanadium oxide $\left(\mathrm{VO}_{2}\right){ }^{[70,72]}$ Recently, a unique sandwiched PAni/graphene/PAni composite paper electrode was fabricated that achieved desirable properties such as high electrical conductivity of $340 \mathrm{~S} / \mathrm{cm}$, lightweight, while retaining good mechanical properties. ${ }^{[70]}$ Lee et al. ${ }^{[72]}$ suggested a method of integrating large sheets of graphene oxide and $\mathrm{VO}_{2}$ nano-belts that greatly reduces the sheet 

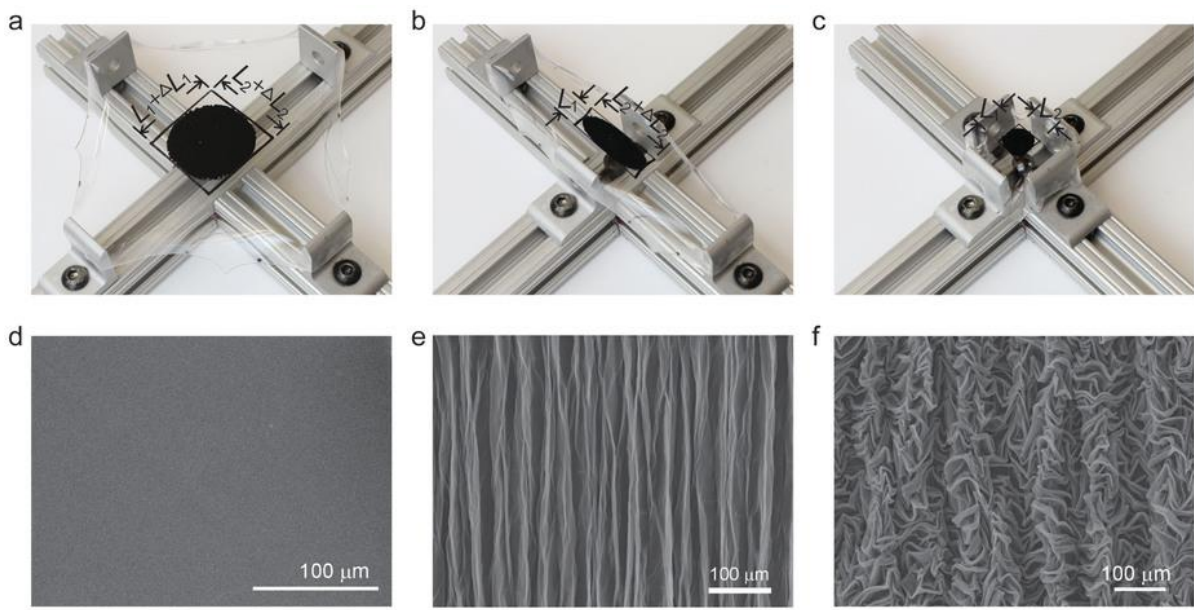

electrolyte systems, these flexible paper electrodes provide an attractive method of creating all-solid-state flexible lightweight SC cells that will potentially revolutionize the future of energy storage. However, the challenge comes with the fabrication of hybrid paper electrodes that allows for high rate capabilities while ensuring the high energy density. There is still space for improvement when it comes to the energy density of paperbased pliable SC electrode.

Figure 11. (a-c) The method for creating crumbled graphene papers. (d-f) SEM images of patterns in the graphene paper. (d) The flat surface of flat graphene paper. (e) When the film was relaxed uniaxially, clear parallel ridges were formed. (f) After the film was stretched and relaxed biaxially and crumpled patterns formed. Reproduced with permission. ${ }^{[71]}$ Copyright 2014, Nature Publishing Group.

\subsection{Flexible Substrate Supported Electrodes}

Research and development in the field of smart textiles have resistance of the electrode, thus improving the overall electrode performance.

Various fabrication methods aimed at increasing charge storage for paper electrodes have been reported. For example, Sun et al. ${ }^{[73]}$ successfully fabricated flexible graphene paper fabricated via a reduction process for SC electrodes induced by flame and have achieved a high surface area of $274.9 \mathrm{~m}^{2} / \mathrm{g}$ that can facilitate ion transfer within the electrode structure. Zhao et al. ${ }^{[74]}$ created porous graphene paper electrode by producing in-plane vacancy defects in the graphene sheets, thus allowing electrolytic ions to efficiently transport within the layered graphene systems. This method utilizes ultrasonication to create high temperature cavitation bubbles that attacks and penetrate the surface to create facile defects. ${ }^{[74]}$ Even though this method has only been applied for battery applications, it is yet to be implemented for SC applications. Wang et al. ${ }^{[75]}$ recently introduced a type of flexible graphene paper that utilized carbon black NPs as pillars. The carbon black particles served as spacers that prevents graphene layer from restacking; this way, the maximum exposed active surface can be ensured. Even at a high scanning rate of 500 $\mathrm{mV} / \mathrm{s}$, the specific capacitance was found to be a reasonable138 F/gin aqueous electrolytes with only a $3.85 \%$ degradation after 2000 test cycles. ${ }^{[75]}$

With the increasing research efforts on paper-based electrodes for SCs, this type of electrodes has shown great promise in producing scalable, low-cost, flexible, high performance energy storage products. The major advantages delivered by this type of paper electrode are namely the sustained electrical conductivity, the mechanical strength and elasticity, as well as the increased availability of active surfaces through the utilization of spacer NPs. ${ }^{[70-71,73-76]}$ When combined with gel-based triggered research interests in fabric based electrodes for SCs that can be integrated with sensors and actuators smart textiles. In many studies, various fabric materials were utilized as the flexible substrate, and then by applying a charge storage coating on the fabric surface in order to enable the functionalities of the electrode. Liu et al. ${ }^{[77]}$ recently used a simple "brush-coating and drying" process to coat a thin layer of graphene oxide on cotton fabric sheets, which obtained a satisfactory $81.7 \mathrm{~F} / \mathrm{g}$ as the specific capacitance. The cotton fabric sheets ensured the flexibility of electrode and provided a facilitated ionic transport platform for efficient electrode/electrolyte interactions. In another study, PPy nanorods were coated onto the cotton fabric via an in-situ polymerization process. The PPy coated cotton fabric electrode achieved very high specific capacitance of $325 \mathrm{~F} / \mathrm{g}$ but suffered from high cycling losses after only 500 cycles. ${ }^{[78]}$ Even though cotton fabric sheets have been researched extensively as a flexible substrate, it does not actively contribute to the overall electrode performance. The electrical conductivity of the electrode material can be significantly improved via the use of graphene fabrics. Zang et al. ${ }^{[79]}$ reported a hybrid electrode with PAni coated on a graphene woven fabric material. The flexibility was ensured while the specific area capacitance reached $23 \mathrm{mF} / \mathrm{cm}^{2}$, a 12 time increase from with the graphene fabric alone. The composite showed ideal cycling stability with $100 \%$ capacitance retention rate after 2000 cycles.

It was deduced that the significant charge storage enhancements originate from the surface area increases and improved facilitation of ion transfer within the electrode's networked 3D structure. And therefore, foams of various types have been considered extensively to be employed as SC electrodes in the recent years. Meng et al. ${ }^{[80]}$ prepared 
a highly pliable porous graphene free-standing composite electrode by growing PAni nanowire arrays on a 3D graphene scaffold surface (Figure 12). The porous graphene structure was created using $\mathrm{CaCO}_{3}$ as a sacrificial template and reducing graphene oxide. The 3D graphene/PAni nanowire electrode demonstrated superior electrochemical performance of up to $385 \mathrm{~F} / \mathrm{g}$, while retaining $90 \%$ of charge storage capacity after 5000 cycles. Graphene foam created via chemical vapor deposition (CVD) coated with polypyrrole obtained specific capacitance of $660 \mathrm{~F} / \mathrm{g}$, and a specific energy of 71 $\mathrm{Wh} / \mathrm{kg}{ }^{[81]}$ The ideal SC electrode behavior was attributed to the 3D interconnected networks of the composite foam structures.

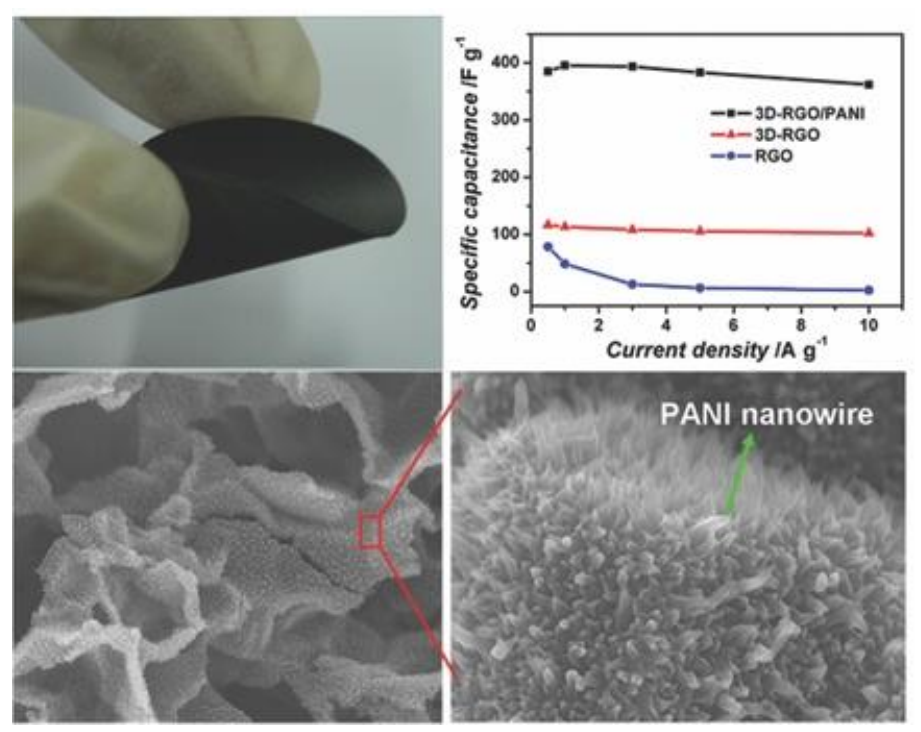

Figure 12. 3D reduced graphene oxide/PAni composite electrode sample with SEM images of the 3D graphene nanostructures showing the grown PAni nanowires. The specific capacitance was not heavily affected by the varying current densities, demonstrating good charge storage capabilities even at high discharge currents. Reproduced with permission. ${ }^{[80]}$ Copyright 2013, John Wiley \& Sons.

MWCNT has also been grown onto a graphene foam to increase the specific surface area without adversely affecting the cycling capability via the introduction of pseudocapacitive materials. ${ }^{[82]}$ The few-layered graphene/MWCNT foam made by Wang et al. ${ }^{[22 \mathrm{a}]}$ is a densely packed hierarchical nanostructure with a high specific surface area of $743 \mathrm{~m}^{2} / \mathrm{g}$, which generated a 233 F/g specific capacitance.

In order to ensure the ions are capable of travelling through the active network, as well as allowing the maximum active surface to be exposed, it was thought that a core-shell structural network involving nano-sized fibers and active conductive polymer coating can be fabricated to optimize SC electrode performance. Herein, we present a novel strategy that disperses graphene nanoplatelets
(GnPs) uniformly and well-adhered to electrospun polyethylene terephthalate (PET) fibers for an efficient access by the solvated ions to interact with the large exposed active surface area. SEM micrograph revealed core-shell structure formation of layered GnPs on top of the PET nanofibers. The average diameter of the pure electrospun PET nanofibers is $114 \pm 41.3 \mathrm{~nm}$, with the GnPs shell of 10-30 nm in thickness, as determined by the SEM image analysis. The specific capacitance has been measured to be $72.1 \mathrm{~F} / \mathrm{g}$ at a relatively fast $\mathrm{CV}$ scan rate of $100 \mathrm{mV} / \mathrm{s}$, based on the weight of the entire electrode, including both GnPs shell and PET substrate. This indicates that the large exposed carbon surface area to the solvated ions within the aqueous electrolyte in the SC cell has reduced the charge transfer resistance at the interface to only $0.4 \Omega$, indicating efficient charge transfer taking place. Galvanic charge/discharge (GCD) tests showed the IR drop at the start of discharge cycles is minimal. Cycling tests verified the cycling stability of the electrodes fabricated from GnPs embedded PET fibers (PET@GnPs). The specific capacitance was measured to be 70.7 F/g after cycling 1000 times, corresponding to a 98.1\% capacitance retention rate. The CV performed at relatively high scan rate showed that the specific capacitance did not dramatically decrease even at $100 \mathrm{mV} / \mathrm{s}$ scan rate, and the cyclic voltammetry still retained an ideal shape even at a very high scan rate of $1000 \mathrm{mV} / \mathrm{s}$. The resulting energy density with a voltage window of $1.2 \mathrm{~V}$ is calculated to be $14.41 \mathrm{Wh} / \mathrm{kg}$, and the power density is $1.16 \mathrm{~kW} / \mathrm{kg}$. The energy density for the PET@GnPs fibers is comparable to that of Ni metal hydride battery but with a higher power density.

The electrolyte used for the testing is aqueous $1 \mathrm{M} \quad \mathrm{H}_{2} \mathrm{SO}_{4} \quad$ electrochemical impedance spectroscopy (EIS) was performed from a high frequency of $10^{5} \mathrm{~Hz}$ to a low frequency of $0.02 \mathrm{~Hz}$. The GCD was performed at a current density of 1 $\mathrm{A} / \mathrm{g}$, with the voltage cycling between $0-1 \mathrm{~V}$. The PET nanofibers were first fabricated via a conventional electrospinning method, as shown in Figure 13a. The electrospinning solution was made with $10 \mathrm{wt} . \%$ PET granular pellets dissolved in 1:1 Dichloromethane (DCM): Trifluoroacetic acid (TFA) solution. The process was carried out for 30 minutes to obtain a very strong, yet flexible thin white PET electrospun film (Figure 13d). PET electrospun fiber film was then placed into the GnPs colloidal suspension to be treated with gentle ultrasonication to open up the porous structure in order to allow infusion of the dispersed GnPs. A black, wetted PET@GnPs film was obtained. The PET@GnPs composite fiber was then taken out of the suspension and washed repeatedly to remove an excess large agglomerates of GnPs attached on the surface of the PET nanofiber. SEM analysis of the pure electrospun PET flexible film showed 

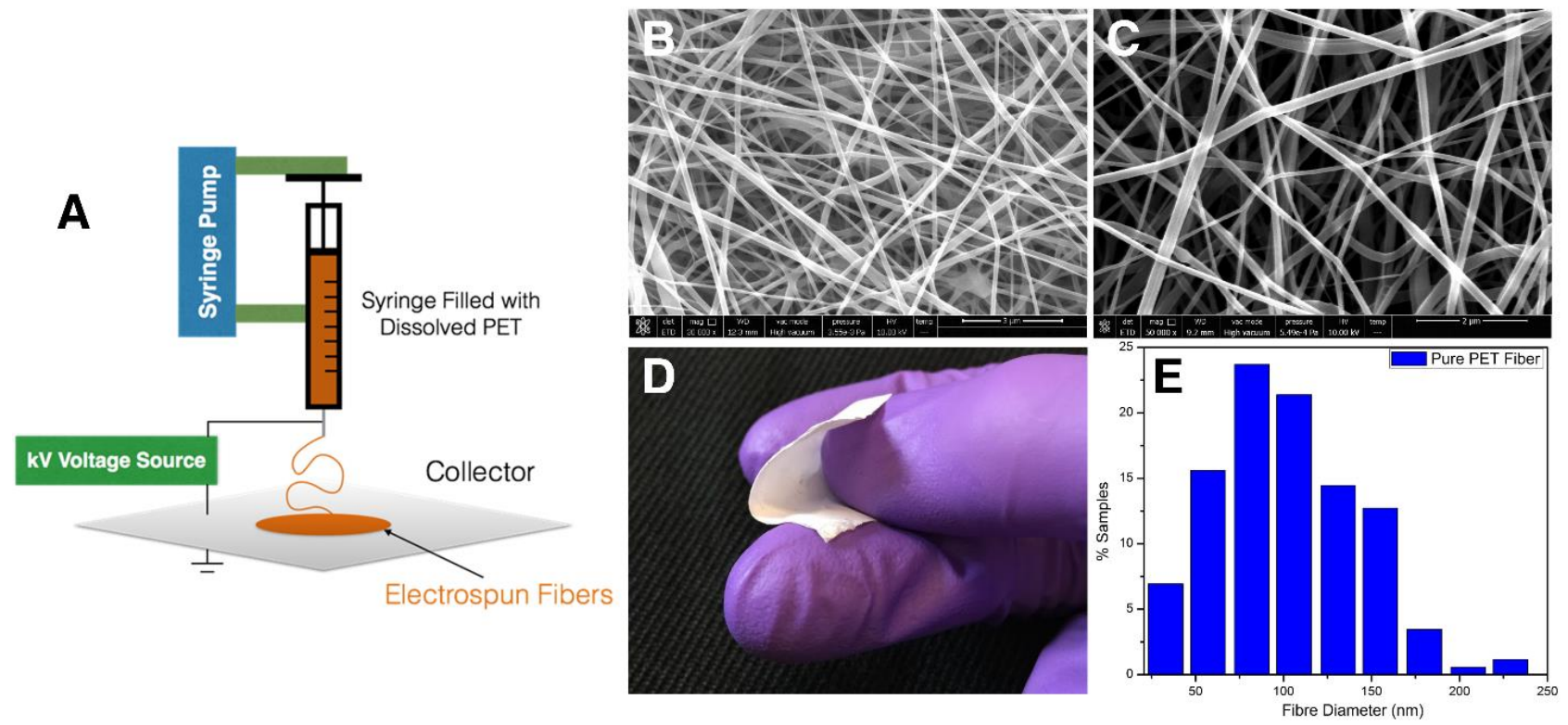

Figure 13. (a) Electrospinning setup used in the PET nanofiber film preparation. (b, c) Scanning electron microscopy of pure electrospun PET film, at different magnification, with a scale bar of $3 \mu \mathrm{m}$ and $2 \mu \mathrm{m}$. (d) Actual flexible pure PET substrate as fabricated. (e) Diameter distribution of PET electrospun nanofibers with $~ 500$ samples using image analysis tools.

characteristics of nano-sized fibers in the diameters ranging from $32.7 \mathrm{~nm}$ to $254 \mathrm{~nm}$, with an average diameter of $114 \pm 41.3 \mathrm{~nm}$ (Figure 13b, $\mathbf{c}, \mathbf{e})$. The flexibility of the actual sample was demonstrated in Figure 13d. Previous studies focus mostly on the surface modification on the electrode material in order to increase the surface area that allows more effective EDLC for charge storage. However, it is noted in our study that to obtain higher surface area it is more effective to construct open-cell porous template structures that allows the solvated ions to penetrate through and reach the interconnected fibers surfaces to achieve improved EDLC.

With larger accessible platform for charge storage, it was then possible to coat a very thin layer of active EDLC material, such as GnPs, on top of the 3D fibrous structure to increase the overall SC performance. However, even with the GnPs particles dispersed by ultrasonication, simply dipping the PET nanofiber into the GnPs colloidal suspension did not allow the GnPs particles to adhere onto the nanofibers, resulting in large agglomerates on the surface (Figure 14a).

As investigated previously, the interfacial adhesion between flat PET film and the monolayer graphene has been found to be poor. ${ }^{\left[{ }^{[3]}\right.}$ And it was also reported that graphene interfacial adhesion to flat PET roll is low in strength and therefore can be used for continuous printing or patterning onto other better adhered substrates. ${ }^{[84]}$ The main mechanism that drives $\mathrm{GnPs}$ to attach to the surface of substrates is via Van der Waal's interactions. However, it was observed that the interaction was poor, and the GnPs were more favored to form large agglomerates rather than adhering to the PET nanofiber surface. Large GnPs agglomerates of sizes in the range of $1-2 \mu \mathrm{m}$ were observed throughout the PET substrate surface. With the ultrasoundassisted fabrication technique, as shown in Figure 14b, a unique lightweight, flexible, high surface area and wellattached PET@GnPs has been successfully created. By placing the PET nanofiber mat into the colloidal solution and place the ultrasonication probe directly above the substrate material. With a gentle sonication, the GnPs particles was observed to attach much better onto the nanofibers to form layers of thickness ranging from 6-16 $\mathrm{nm}$ after repeated washing, with an average of $10 \pm 4.2 \mathrm{~nm}$. GnPs have a typical thickness of 8-15 nm which correspond well to the thickness increase observed experimentally. The stacked GnPs allowed for gaps that resembled a viable mesoporous structures (as hypothesized from the low charge transfer resistance in the EIS analysis), which was shown to be the most effective porous structure for the ions to move freely while the specific surface area is optimized. ${ }^{[56]}$ Therefore, even though the layered effect still exists with the GnPs, the energy and power density performance of the electrode is still considerably higher than other types of graphene-based SCs. ${ }^{[57 a, 85]}$ The thickness of the obtained PET@GnPs film was controlled at $0.15 \pm 0.01 \mathrm{~mm}$. The adhered GnPs particles on the PET nano-substrate accounted for $18.22 \%$ of the total weight of the PET@GnPs fiber according to TGA analysis. The superflex PET@GnPs electrodes allow the SC cell to be highly flexible. The flexibility test has showed that the 

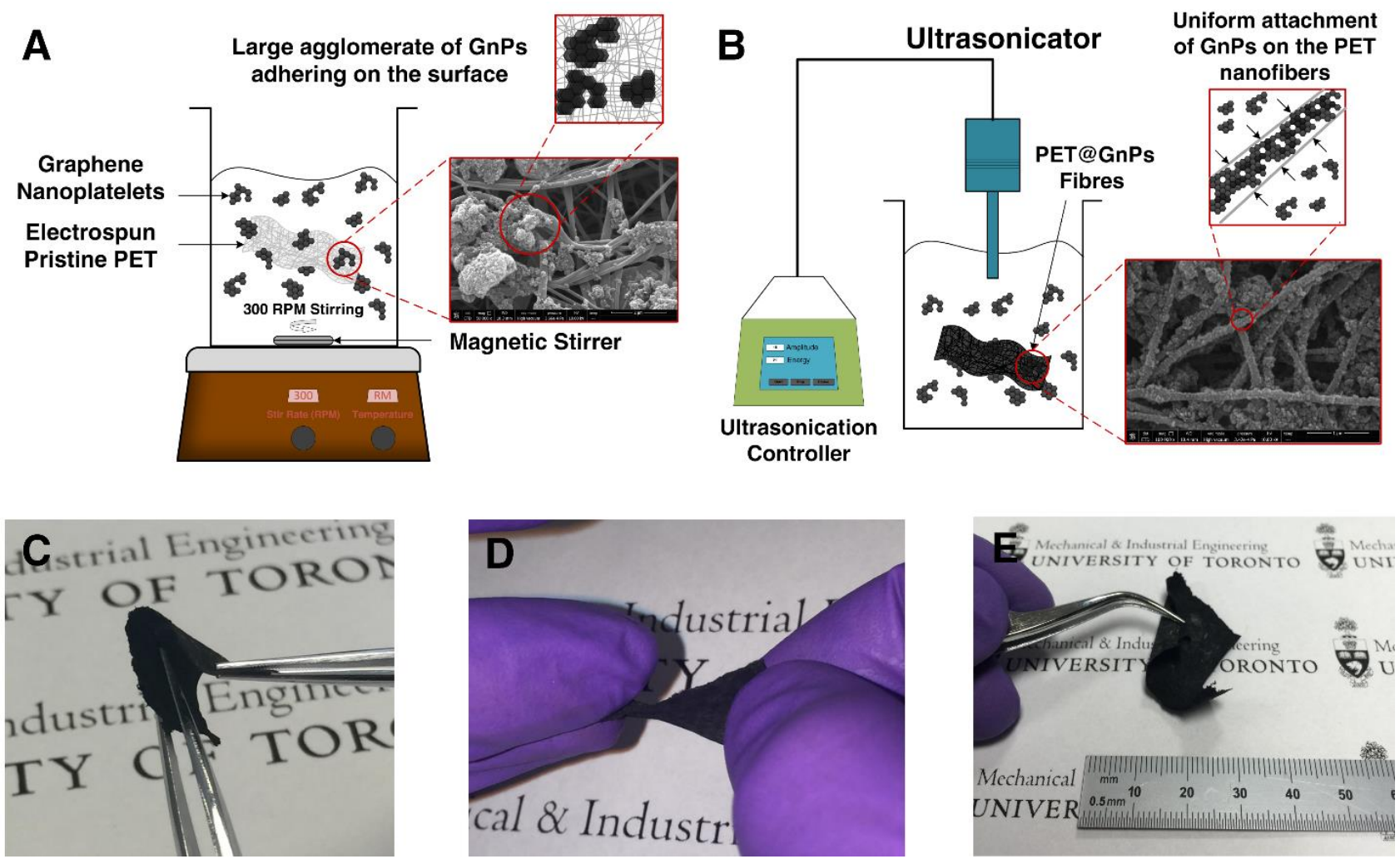

Figure 14. (a) Large agglomerates formation on top of the PET film surface after dipping in ultrasound-dispersed GnPs suspension. (b) Ultrasonication-assisted method to induce better adhered GnPs on the PET surface. The average thickness of the coating was measured to be $10 \pm 4.2 \mathrm{~nm}$. (c-e) Flexibility testing showing the PET@GnPs fibers' ability to bend or twist without permanent strain deformations.

PET@GnPs fiber mat can be bent $360^{\circ}$ or twisted one complete turn without exhibiting irreversible strain deformations (Figure 14c-e).

$\mathrm{CV}$ performed at various scan rates ranging from 5 $\mathrm{mV} / \mathrm{s}$ to $1000 \mathrm{mV} / \mathrm{s}$ has shown that the charge transfer takes place effectively within the electrode. As shown in Figure 15a, the rectangular shape of the $\mathrm{CV}$ was retained even at very high scan rates (i.e. $1000 \mathrm{mV} / \mathrm{s}$ ). This indicated that the charge transfer between the electrolyte and the porous electrode was not hindered, and therefore demonstrated an ideally shaped CV graph. The obtained capacitance at $100 \mathrm{mV} / \mathrm{s}$ was $72.1 \mathrm{~F} / \mathrm{g}$, indicating that majority of the GnPs surface area was accessible to the ions in the electrolyte, allowing for very effective charge storage mechanism. We have created a nano-porous fibrous structure that allows the electrolyte to access of the surface area available throughout the PET@GnPs film thickness, thus allowing better EDLC performance. Herein, the porous structures in the PET@GnPs fibers can also be effectively controlled by tuning electrospinning and GnPs sonication-assisted coating parameters. The EIS result shown in Figure 15c indicates that the charge transfer impedance is very low at about $0.4 \Omega$. This shows that even with high scan rates, the $\mathrm{CV}$ graphs still depicted a fairly rectangular and ideal capacitor behavior for the
PET@GnPs electrode, indicating that the electrolytic ions are allowed to travel freely into and out of the porous electrospun PET@GnPs electrode system. From the GCD curves shown in Figure 15d, the energy density was calculated to be $14.41 \mathrm{Wh} / \mathrm{kg}$, and the power density at 1.16 $\mathrm{kW} / \mathrm{kg}$ for the PET@GnPs fibers, based on the entire weight of the electrode. With the utilization of nonaqueous solid-state electrolyte, the voltage window can be stretched from $1.2 \mathrm{~V}$ to $4.0 \mathrm{~V}$, which would result in dramatic increases in both energy and power densities. Therefore, this type of SC electrodes can be suitable for many flexible, high power requirement applications. The cycling test showed a common trend with carbon-based EDL capacitors (Figure 15e, f), where the degradation in specific capacitance is essentially zero in the first 2000 cycles. At 3000 cycles, it was found that the specific capacitance decreased to around $94.2 \%$ of the initial value, which is still considerably higher than most pseudocapacitance materials. It is hypothesized that this reduction may be due to the detachment of GnPs from the PET substrate after 2000 cycles of charge cycles. Surface treatment of the PET fiber scaffold structure may provide a key to further improving the adhesion between the GnPs and the PET nanofibers, allowing better cycling performance of the PET@GnPs superflex electrode 

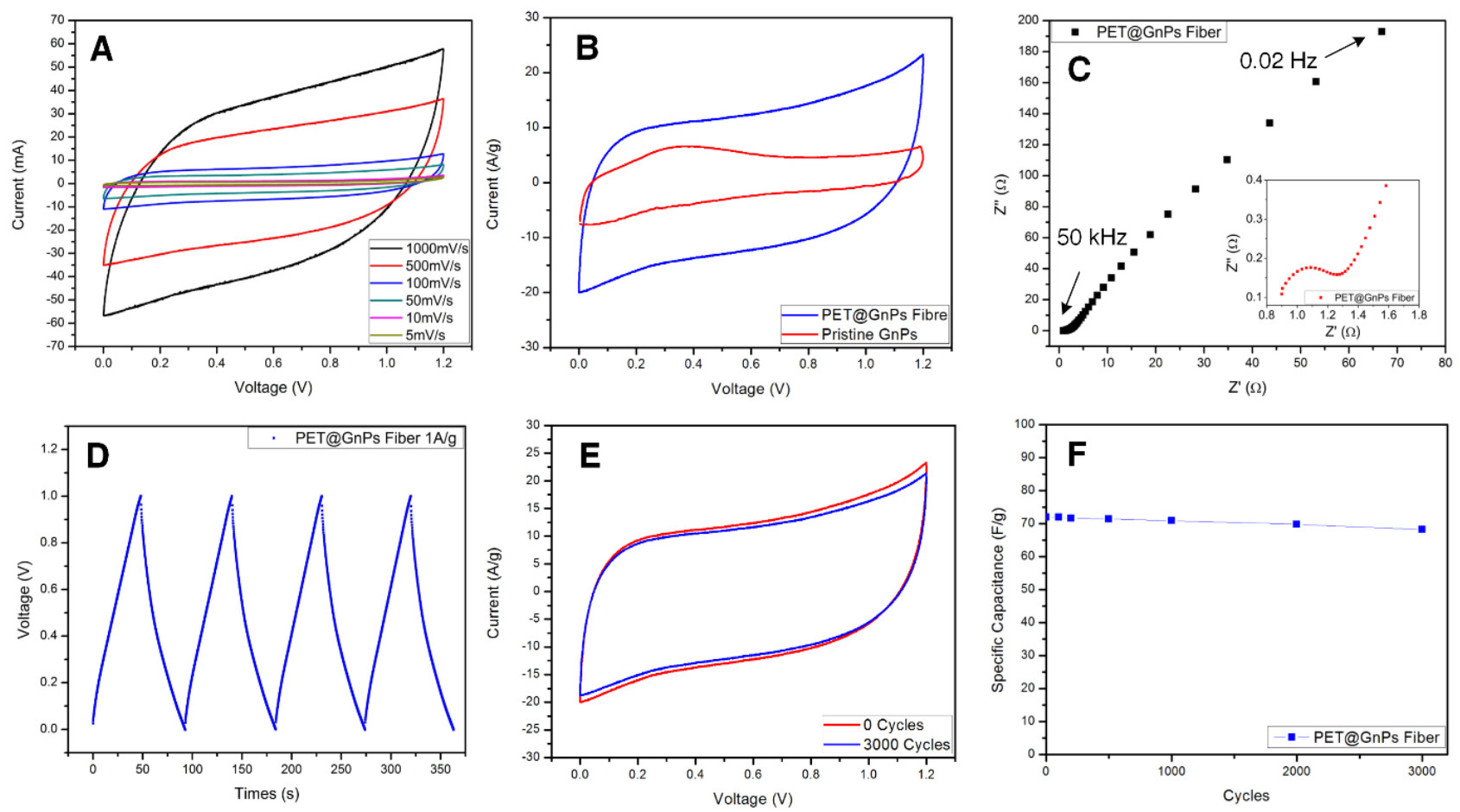

Figure 15. (a) Mostly rectangular CV graphs measured at different scan rates from $1000 \mathrm{mV} / \mathrm{s}$ to $5 \mathrm{mV} / \mathrm{s}$, with ideal capacitor behaviors. (b) A comparison between the pristine GnPs and PET@GnPs electrode in terms of the specific current calculated based on the weight of the entire electrode mass. (c) The Nyquist plot of the EIS measurement of PET@GnPs fibers with an inset of zoomed-in graph showing the charge transfer impedance to be around $0.4 \Omega$. (d) The GCD test of the PET@GnPs fiber electrodes. (e) The CV graph comparison of the electrode cycling after 0 cycles and 3000 cycles, showing very similar performances. (f) The measured specific capacitance drop of the PET@GnPs fibers after 3000 cycles was $5.8 \%$.

systems. This novel ultrasound-assisted fabrication strategy showed promise in creating core-shell structure superflex, high-performance SC devices that can be applicable for energy storage purposes in biomedical or personal electronic industries.

The difficulty associated with electrodes for flexible substrate is the adhesion interactions between the active materials and the dormant substrate. While the substrates typically do not actively participate in the charge storage, they provide a suitable large-surface-area platform for the active electrode materials. If there is poor adhesion between the substrate and the active materials, it may lead to poor cycling capability and decreased shelf-life. In addition, the possible chemical reactions between the substrate material and the electrolyte system can also negatively contribute to the electrode performance, leading to possible increase in degradation rate.

\section{Conclusions and Outlook}

In this Progress Report, recent progress and challenges associated with flexible energy storage systems for the wearable electronics devices are presented. Although, a lot of research has been carried out on pliable electrodes such as in paper based (cellulose paper, textiles, CNT/graphene free standing films) or 3D bulky-compressible materials (hydrogels and graphene or CNT sponge etc.), their performance is still not up to par with their rigid counterparts. The substrate supported electrodes (cellulose paper or textile) have high flexibility, but their energy and power densities are still low. Composite free standing films of graphene or CNT with high capacity materials $(\mathrm{Si}, \mathrm{Ge}$, Sn and metal oxides etc.) improve the electrochemical performance, but possess poor mechanical strength. Another major challenge is to maintain the rate capabilities while increasing the energy and power densities. In the case of flexible SCs with hybrid electrode materials, the energy density is typically ensured by the inclusion of pseudocapacitive components, but that decreases the rate capabilities due to interfacial REDOX reactions. To overcome these problem electrode materials with a high specific surface area and porous structure that facilitates a short ion transfer path and high electronic conductivity are highly desired. A possible solution to improve the electrochemical activity and electronic or ionic conductivity is the $3 \mathrm{D}$ conducting network. Xin et al. ${ }^{[86]}$ reported on 3D porous nanocarbon networks with different carbon morphologies such as (NPs, CNT, graphene and 
nanoporous carbon) that can increase the electrochemical performance by providing a fast electron and ion transfer during charging and discharging. In addition, charge storage capacity can be further increased by hybridizing these 3D nonporous carbon networks with nanostructured electrode materials.

Obtaining stable electrochemical performance under adverse thermo-mechanical stresses in flexible storage devices is highly challenging. Most pliable electrodes under constant mechanical deformations exhibit low performance and this greatly hinders their practical applications. The current technique to address this issue is to hybridize the active materials with CNT or graphenebased substrate.

Packaging is another difficulty that can affect the reliability and the consequence performance of the flexible storage devices. A properly designed and well-assembled packaged device prevents the electrolyte from leaking during bending, stretching, or compressing deformation. This problem can be addressed using solid state electrolytes that should be flexible, ionic conductive and have excellent adhesion with electrode materials during bending, stretching or compressing.

The lack of a good understanding of the electrode/ electrolyte interfacial interactions under mechanical deformations contributes to an insufficient control over the performance of these flexible storage devices. To date, most of research studies focused on reporting experimental observations instead of understanding and predicting their behaviors. Advanced simulation techniques that allow a better understanding of the electrode surface behaviors can lead to improved control over the electrical conductivity, energy storage performance and mechanical properties.

Electrode materials, their design and fabrication are key points to ensure they have the designed flexibility. 3D printing technology can provide cost-effective, large-scale production of practical pliable electrodes. However, the absence of an industry standard to assess variations in the electrochemical performance under varying mechanical deformations is hindering their proper development. Despite the many challenges, the future of flexible LIBs/SCs is extremely bright. The increasingly improved mechanical and electrical properties of pliable electrodes for flexible LIBs/SCs that are low cost and with high energy and power densities will undoubtedly stimulate many new applications in wearable electronic devices.

\section{Acknowledgements}

The authors would like to acknowledge the National Science Foundation of China (NSFC), Natural Sciences and Engineering Research Council of Canada (NSERC), Canada Foundation for Innovation (CFI) and Canada Research Chairs program (CRC) for the financial support they have provided for this research work.

Received: XX, 2016

Revised: XX, 2016 Published online: XX, 2016
[1] a) Z. Liu, J. Xu, D. Chen, G. Shen, Chem. Soc. Rev. 2015 , 44, 161; b) T. Cheng, Y. Zhang, W. Y. Lai, W. Huang, Adv. Mater. 2015, 27, 3349; c) K. D. Harris, A. L. Elias, H. J. Chung, J. Mater. Sci. 2015, 51, 2771; d) J. A. Rogers, T. Someya, Y. Huang, Science 2010, 327, 1603; e) S. Bae, H. Kim, Y. Lee, X. Xu, J.-S. Park, Y. Zheng, J. Balakrishnan, T. Lei, H. Ri Kim, Y. I. Song, Y.-J. Kim, K. S. Kim, B. Ozyilmaz, J.-H. Ahn, B. H. Hong, S. Iijima, Nat. Nano 2010, 5,574

[2] Y. Shao, M. F. El-Kady, L. J. Wang, Q. Zhang, Y. Li, H. Wang, M. F. Mousavi, R. B. Kaner, Chem. Soc. Rev. 2015, 44, 3639.

[3] Z. Wang, Z. Wu, N. Bramnik, S. Mitra, Adv. Mater. 2014, 26, 970.

[4] Z. Wang, N. Bramnik, S. Roy, G. Di Benedetto, J. L. Zunino, S. Mitra, J. Power Sources 2013, 237, 210.

[5] I. Sultana, M. M. Rahman, J. Wang, C. Wang, G. G. Wallace, H.-K. Liu, Electrochim. Acta 2012, 83, 209.

[6] L. Sannier, R. Bouchet, S. Grugeon, E. Naudin, E. Vidal, J. M. Tarascon, J. Power Sources 2005, 144, 231.

[7] Y. Hu, X. Sun, J. Mater. Chem. A 2014, 2, 10712.

[8] G. Xin, Y. Wang, X. Liu, J. Zhang, Y. Wang, J. Huang, J. Zang, Electrochim. Acta 2015, 167, 254.

[9] a) T. Chen, L. Dai, J. Mater. Chem. A 2014, 2, 10756; b) X. Li, B. Wei, Nano Energy 2013, 2, 159; c) S. Shi, C. Xu, C. Yang, J. Li, H. Du, B. Li, F. Kang, Particuology 2013, 11, 371; d) C. Wang, G. G. Wallace, Electrochim. Acta 2015, 175, 87; e) X. Wang, X. Lu, B. Liu, D. Chen, Y. Tong, G. Shen, Adv. Mater. 2014, 26, 4763; f) G. Zhou, F. Li, H.-M. Cheng, Energy Environ. Sci. 2014, 7, 1307.

[10] a) N. Mahmood, Y. Hou, Adv. Sci. 2014, 1, 1400012; b) N. Mahmood, M. Tahir, A. Mahmood, J. Zhu, C. Cao, Y. Hou, Nano Energy 2015, 11, 267; c) A. Mahmood, R. Zou, Q. Wang, W. Xia, H. Tabassum, B. Qiu, R. Zhao, ACS Appl. Mater. Interfaces 2016, 8, 2148; d) N. Mahmood, M. Tahir, A. Mahmood, W. Yang, X. Gu, C. Cao, Y. Zhang, Y. Hou, Sci. China Mater. 2015, 58, 114; e) Q. Yue, H. Jiang, Y. Hu, G. Jia, C. Li, Chem. Commun. 2014, 50, 13362; f) H. Jiang, Y. Hu, S. Guo, C. Yan, P. S. Lee, C. Li, ACS Nano 2014, 8, 6038; g) H. Jiang, G. Jia, Y. Hu, Q. Cheng, Y. Fu, C. Li, Ind. Eng. Chem. Res. 2015, 54, 2960; h) H. Jiang, D. Ren, H. Wang, Y. Hu, S. Guo, H. Yuan, P. Hu, L. Zhang, C. Li, Adv. Mater. 2015, 27, 3687.

[11] H. Gwon, J. Hong, H. Kim, D.-H. Seo, S. Jeon, K. Kang, Energy Environ. Sci. 2014, 7, 538.

[12] L. Wen, F. Li, H.-Z. Luo, H.-M. Cheng, in Nanocarbons for Advanced Energy Storage, Vol. 1, Wiley-VCH Verlag GmbH \& Co. KGaA, 2015, 119.

[13] L. Hu, Y. Cui, Energy Environ. Sci. 2012, 5, 6423.

[14] a) X. Wang, K. Jiang, G. Shen, Mater. Today 2015, 18, 265; b) Y. Zhang, Y. Zhao, J. Ren, W. Weng, H. Peng, Adv. Mater. 2015.

[15] M. Inagaki, H. Konno, O. Tanaike, J. Power Sources 2010 , $195,7880$.

[16] B. A. Johnson, R. E. White, J. Power Sources 1998, 70, 48.

[17] K. Wang, S. Luo, Y. Wu, X. He, F. Zhao, J. Wang, K. Jiang, S. Fan, Adv. Funct. Mater. 2013, 23, 846.

[18] a) D. Tobjork, R. Osterbacka, Adv. Mater. 2011, 23, 1935; b) L. Nyholm, G. Nystrom, A. Mihranyan, M. Stromme, Adv. Mater. 2011, 23, 3751.

[19] a) V. L. Pushparaj, M. M. Shaijumon, A. Kumar, S. 
Murugesan, L. Ci, R. Vajtai, R. J. Linhardt, O. Nalamasu, P. M. Ajayan, Proc. Natl. Acad. Sci. U. S. A. 2007, 104, 13574; b) Y. R. Kang, Y. L. Li, F. Hou, Y. Y. Wen, D. Su, Nanoscale 2012, 4, 3248; c) L. Hu, N. Liu, M. Eskilsson, G. Zheng, J. McDonough, L. Wågberg, Y. Cui, Nano Energy 2013, 2, 138.

[20] L. Hu, J. W. Choi, Y. Yang, S. Jeong, F. La Mantia, L. F. Cui, Y. Cui, Proc. Natl. Acad. Sci. U. S. A. 2009, 106, 21490.

[21] a) J. Wang, L. Li, C. L. Wong, S. Madhavi, Nanotechnol. 2012, 23, 495401; b) Q. Cheng, Z. Song, T. Ma, B. B. Smith, R. Tang, H. Yu, H. Jiang, C. K. Chan, Nano Lett. 2013, 13, 4969.

[22] L. Hu, M. Pasta, F. L. Mantia, L. Cui, S. Jeong, H. D. Deshazer, J. W. Choi, S. M. Han, Y. Cui, Nano Lett. 2010, 10,708 .

[23] L. Hu, F. La Mantia, H. Wu, X. Xie, J. McDonough, M. Pasta, Y. Cui, Adv. Energy Mater. 2011, 1, 1012.

[24] L. Hu, H. Wu, F. La Mantia, Y. Yang, Y. Cui, ACS Nano 2010, 4, 5843.

[25] A. M. Gaikwad, B. V. Khau, G. Davies, B. Hertzberg, D. A. Steingart, A. C. Arias, Adv. Energy Mater. 2015, 5, 1401389

[26] a) Z. Song, T. Ma, R. Tang, Q. Cheng, X. Wang, D. Krishnaraju, R. Panat, C. K. Chan, H. Yu, H. Jiang, Nat. Commun. 2014, 5, 3140; b) G. Kettlgruber, M. Kaltenbrunner, C. M. Siket, R. Moser, I. M. Graz, R. Schwödiauer, S. Bauer, J. Mater. Chem. A 2013, 1, 5505.

[27] a) H. Gwon, H.-S. Kim, K. U. Lee, D.-H. Seo, Y. C. Park, Y.-S. Lee, B. T. Ahn, K. Kang, Energy Environ. Sci. 2011, 4, 1277; b) Y. Shi, L. Wen, G. Zhou, J. Chen, S. Pei, K. Huang, H.-M. Cheng, F. Li, 2D Mater. 2015, 2, 024004.

[28] a) R. S. Morris, B. G. Dixon, T. Gennett, R. Raffaelle, M. J. Heben, J. Power Sources 2004, 138, 277; b) S. Y. Chew, S. H. Ng, J. Wang, P. Novák, F. Krumeich, S. L. Chou, J. Chen, H. K. Liu, Carbon 2009, 47, 2976; c) X. Li, J. Yang, Y. Hu, J. Wang, Y. Li, M. Cai, R. Li, X. Sun, J. Mater. Chem. 2012, 22, 18847; d) J. Chen, A. I. Minett, Y. Liu, C. Lynam, P. Sherrell, C. Wang, G. G. Wallace, Adv. Mater. 2008, 20, 566; e) J. Chen, Y. Liu, A. I. Minett, C. Lynam, J. Wang, G. G. Wallace, Chem. Mater. 2007, 19, 3595; f) S. Yoon, S. Lee, S. Kim, K.-W. Park, D. Cho, Y. Jeong, J. Power Sources 2015, 279, 495; g) C. Wang, D. Li, C. O. Too, G. G. Wallace, Chem. Mater. 2009, 21, 2604; h) H. Bi, J. Chen, W. Zhao, S. Sun, Y. Tang, T. Lin, F. Huang, X. Zhou, X. Xie, M. Jiang, $R S C A d v$. 2013, 3, 8454; i) Y. $\mathrm{Hu}, \mathrm{X}$. Li, D. Geng, M. Cai, R. Li, X. Sun, Electrochim. Acta 2013, 91, 227.

[29] a) J. Xu, X. Wei, J. Cao, Y. Dong, G. Wang, Y. Xue, W. Wang, Z. Chen, Electrochim. Acta 2015, 152, 391; b) Y. Hu, X. Li, J. Wang, R. Li, X. Sun, J. Power Sources 2013, 237, 41; c) K. Shu, C. Wang, S. Li, C. Zhao, Y. Yang, H. Liu, G. Wallace, J. Mater. Chem. A 2015, 3, 4428.

[30] a) L. Yue, H. Zhong, L. Zhang, Electrochim. Acta 2012, 76, 326; b) J. K. Lee, K. B. Smith, C. M. Hayner, H. H. Kung, Chem. Commun. 2010, 46, 2025; c) I. Elizabeth, R. B. Mathur, P. H. Maheshwari, B. P. Singh, S. Gopukumar, Electrochim. Acta 2015, 176, 735; d) Z.-S. Wu, G. Zhou, L.-C. Yin, W. Ren, F. Li, H.-M. Cheng, Nano Energy 2012, 1, 107; e) H.-C. Tao, L.-Z. Fan, Y. Mei, X. Qu, Electrochem. Commun. 2011, 13, 1332; f) B. Wang, X. Li, B. Luo, Y. Jia, L. Zhi, Nanoscale 2013, 5, 1470; g) R. A.
DiLeo, S. Frisco, M. J. Ganter, R. E. Rogers, R. P. Raffaelle, B. J. Landi, J. Phys. Chem. C 2011, 115, 22609; h) X. Jia, Z. Chen, X. Cui, Y. Peng, X. Wang, G. Wang, F. Wei, Y. Lu, ACS Nano 2012, 6, 9911; i) B. Wang, X. Li, X. Zhang, B. Luo, M. Jin, M. Liang, S. A. Dayeh, S. T. Picraux, L. Zhi, ACS Nano 2013, 7, 1437; j) M. Dirican, O. Yildiz, Y. Lu, X. Fang, H. Jiang, H. Kizil, X. Zhang, Electrochim. Acta 2015, 169, 52; k) L. Noerochim, J.-Z. Wang, S.-L. Chou, D. Wexler, H.-K. Liu, Carbon 2012, 50, 1289 ; 1) H. Köse, Ş. Karaal, A. O. Aydın, H. Akbulut, $J$. Power Sources 2015, 295, 235; m) N. Li, G. Zhou, R. Fang, F. Li, H. M. Cheng, Nanoscale 2013, 5, 7780; n) X. Yang, K. Fan, Y. Zhu, J. Shen, X. Jiang, P. Zhao, S. Luan, C. Li, ACS Appl Mater Interfaces 2013, 5, 997; o) Y. S. Yun, J. M. Kim, H. H. Park, J. Lee, Y. S. Huh, H.-J. Jin, J. Power Sources 2013, 244, 747; p) C. Ban, Z. Wu, D. T. Gillaspie, L. Chen, Y. Yan, J. L. Blackburn, A. C. Dillon, Adv. Mater. 2010, 22, E145; q) R. Wang, C. Xu, J. Sun, L. Gao, C. Lin, J. Mater. Chem. A 2013, 1, 1794; r) G. Zhou, D.-W. Wang, P.-X. Hou, W. Li, N. Li, C. Liu, F. Li, H.-M. Cheng, J. Mater. Chem. 2012, 22, 17942; s) Y. E. Miao, Y. Huang, L. Zhang, W. Fan, F. Lai, T. Liu, Nanoscale 2015, 7, 11093; t) L. Zhang, Y. Huang, Y. Zhang, W. Fan, T. Liu, ACS Appl Mater Interfaces 2015, 7, 27823; u) L. Zhang, W. Fan, T. Liu, RSC Adv. 2015, 5, 43130.

[31] C. Ma, C. Ma, J. Wang, H. Wang, J. Shi, Y. Song, Q. Guo, L. Liu, Carbon 2014, 72, 38.

[32] Y. Zhao, C. Ma, Y. Li, H. Chen, Z. Shao, Carbon 2015, 95, 494.

[33] S. Liu, R. Wang, M. Liu, J. Luo, X. Jin, J. Sun, L. Gao, J. Mater. Chem. A 2014, 2, 4598.

[34] Z. Zhang, Q. Tan, Z. Zhong, F. Su, RSC Adv. 2015, 5, 40018.

[35] a) Z. Chen, W. Ren, L. Gao, B. Liu, S. Pei, H.-M. Cheng, Nat. Mater. 2011, 10, 424; b) X. Gui, J. Wei, K. Wang, A. Cao, H. Zhu, Y. Jia, Q. Shu, D. Wu, Adv. Mater. 2010, 22, 617; c) S. Jing, H. Jiang, Y. Hu, J. Shen, C. Li, Adv. Funct. Mater. 2015, 25, 5395.

[36] a) S. Nardecchia, D. Carriazo, M. L. Ferrer, M. C. Gutierrez, F. del Monte, Chem Soc Rev 2013, 42, 794; b) L. Liu, W. Ma, Z. Zhang, Small 2011, 7, 1504.

[37] X. Huang, X. Qi, F. Boey, H. Zhang, Chem Soc Rev 2012 , $41,666$.

[38] a) B. Liu, J. Zhang, X. Wang, G. Chen, D. Chen, C. Zhou, G. Shen, Nano Lett. 2012, 12, 3005; b) B. Liu, X. Wang, B. Liu, Q. Wang, D. Tan, W. Song, X. Hou, D. Chen, G. Shen, Nano Res. 2013, 6, 525; c) X. Wei, W. Li, J. A. Shi, L. Gu, Y. Yu, ACS Appl Mater Interfaces 2015, 7, 27804; d) B. Wang, S. Li, X. Wu, B. Li, J. Liu, M. Yu, Phys. Chem. Chem. Phys. 2015, 17, 21476; e) X. Hou, B. Liu, X. Wang, Z. Wang, Q. Wang, D. Chen, G. Shen, Nanoscale 2013, 5, 7831; f) W. Li, X. Wang, B. Liu, J. Xu, B. Liang, T. Luo, S. Luo, D. Chen, G. Shen, Nanoscale 2013, 5, 10291; g) C. Wang, W. Wan, Y. Huang, J. Chen, H. H. Zhou, X. X. Zhang, Nanoscale 2014, 6, 5351; h) X. Hou, X. Wang, B. Liu, Q. Wang, T. Luo, D. Chen, G. Shen, Nanoscale 2014, 6, 8858; i) X. Wang, L. Sun, R. A. Susantyoko, Q. Zhang, Carbon 2016, 98, 504; j) X. H. Wang, C. Guan, L. M. Sun, R. A. Susantyoko, H. J. Fan, Q. Zhang, J. Mater. Chem. A 2015, 3, 15394; k) M.-S. Balogun, Z. Wu, Y. Luo, W. Qiu, X. Fan, B. Long, M. Huang, P. Liu, Y. Tong, J. Power Sources 2016, 308, 7; 1) G. Zhang, S. Hou, H. Zhang, W. 
Zeng, F. Yan, C. C. Li, H. Duan, Adv. Mater. 2015, 27, 2400; m) H. Yu, C. Zhu, K. Zhang, Y. Chen, C. Li, P. Gao, P. Yang, Q. Ouyang, J. Mater. Chem. A 2014, 2, 4551.

[39] X. Cao, Y. Shi, W. Shi, X. Rui, Q. Yan, J. Kong, H. Zhang, Small 2013, 9, 3433.

[40] Z. C. Na Li, Wencai Ren, Feng Li, and Hui-Ming Cheng, Proc. Natl. Acad. Sci. U. S. A. 2012, 109, 17360.

[41] a) P. Li, Y. Yang, E. Shi, Q. Shen, Y. Shang, S. Wu, J. Wei, K. Wang, H. Zhu, Q. Yuan, A. Cao, D. Wu, ACS Appl Mater Interfaces 2014, 6, 5228; b) B. Luo, T. Qiu, B. Wang, L. Hao, X. Li, A. Cao, L. Zhi, Nanoscale 2015, 7, 20380; c) L. Li, R. Li, S. Gai, P. Gao, F. He, M. Zhang, Y. Chen, P. Yang, J. Mater. Chem. A 2015, 3, 15642; d) X. Cheng, X. Gui, Z. Lin, Y. Zheng, M. Liu, R. Zhan, Y. Zhu, Z. Tang, J. Mater. Chem. A 2015, 3, 20927; e) X. Chen, H. Zhu, Y.-C. Chen, Y. Shang, A. Cao, L. Hu, G. W. Rubloff, ACS Nano 2012, 6, 7948; f) Z. Ma, Y. Wang, Y. Yang, M. Yousaf, M. Zou, A. Cao, R. P. S. Han, RSC Adv. 2016, 6, 30098.

[42] J. Wang, J. Liu, D. Chao, J. Yan, J. Lin, Z. X. Shen, Adv. Mater. 2014, 26, 7162 .

[43] W. Zeng, L. Shu, Q. Li, S. Chen, F. Wang, X. M. Tao, Adv. Mater. 2014, 26, 5310.

[44] D. Liu, M. Zhao, Y. Li, Z. Bian, L. Zhang, Y. Shang, X. Xia, S. Zhang, D. Yun, Z. Liu, A. Cao, C. Huang, ACS Nano 2012, 6, 11027.

[45] T. Yamada, Y. Hayamizu, Y. Yamamoto, Y. Yomogida, A. Izadi-Najafabadi, D. N. Futaba, K. Hata, Nat. Nano 2011, 6, 296.

[46] a) J. Ren, L. Li, C. Chen, X. Chen, Z. Cai, L. Qiu, Y. Wang, X. Zhu, H. Peng, Adv. Mater. 2013, 25, 1155; b) H. Lin, W. Weng, J. Ren, L. Qiu, Z. Zhang, P. Chen, X. Chen, J. Deng, Y. Wang, H. Peng, Adv. Mater. 2014, 26, 1217; c) C.-F. Sun, H. Zhu, E. B. Baker Iii, M. Okada, J. Wan, A. Ghemes, Y. Inoue, L. Hu, Y. Wang, Nano Energy 2013, 2, 987.

[47] Y. H. Kwon, S. W. Woo, H. R. Jung, H. K. Yu, K. Kim, B. H. Oh, S. Ahn, S. Y. Lee, S. W. Song, J. Cho, H. C. Shin, J. Y. Kim, Adv. Mater. 2012, 24, 5192.

[48] W. Weng, Q. Sun, Y. Zhang, H. Lin, J. Ren, X. Lu, M. Wang, H. Peng, Nano Lett. 2014, 14, 3432.

[49] J. Ren, Y. Zhang, W. Bai, X. Chen, Z. Zhang, X. Fang, W. Weng, Y. Wang, H. Peng, Angew. Chem., Int. Ed. Engl. 2014, 53, 7864.

[50] a) S. Xu, Y. Zhang, J. Cho, J. Lee, X. Huang, L. Jia, J. A. Fan, Y. Su, J. Su, H. Zhang, H. Cheng, B. Lu, C. Yu, C. Chuang, T. I. Kim, T. Song, K. Shigeta, S. Kang, C. Dagdeviren, I. Petrov, P. V. Braun, Y. Huang, U. Paik, J. A. Rogers, Nat. Commun. 2013, 4, 1543; b) Y. Zhang, W. Bai, J. Ren, W. Weng, H. Lin, Z. Zhang, H. Peng, J. Mater. Chem. A 2014, 2, 11054.

[51] Y. Zhang, W. Bai, X. Cheng, J. Ren, W. Weng, P. Chen, X. Fang, Z. Zhang, H. Peng, Angew. Chem., Int. Ed. Engl. 2014, 53, 14564.

[52] a) F. Béguin, V. Presser, A. Balducci, E. Frackowiak, $A d v$. Mater. 2014, 26, 2283; b) P. Simon, Y. Gogotsi, Nat. Mater. 2008, 7, 845; c) B. E. Conway, W. G. Pell, J. Solid State Electrochem. 2003, 7, 637.

[53] A. Vlad, N. Singh, J. Rolland, S. Melinte, P. M. Ajayan, J. F. Gohy, Sci. Rep. 2014, 4.

[54] a) Y. Meng, Y. Zhao, C. Hu, H. Cheng, Y. Hu, Z. Zhang, G. Shi, L. Qu, Adv. Mater. 2013, 25, 2326; b) Q. Niu, K. Gao, Z. Shao, Nanoscale 2014, 6, 4083.
[55] C. Meng, O. Z. Gall, P. P. Irazoqui, Biomed. Microdevices 2013, 15, 973.

[56] B. E. Conway, Electrochemical supercapacitors: scientific fundamentals and technological applications, Kluwer Academic/Plenum Publishers, New York 1999.

[57] a) M. D. Stoller, S. Park, Y. Zhu, J. An, R. S. Ruoff, Nano Lett. 2008, 8, 3498; b) C. Emmenegger, P. Mauron, P. Sudan, P. Wenger, V. Hermann, R. Gallay, A. Züttel, J. Power Sources 2003, 124, 321; c) T. Osaka, X. Liu, M. Nojima, T. Momma, J. Electrochem. Soc. 1999, 146, 1724.

[58] X. Wang, X. Lu, B. Liu, D. Chen, Y. Tong, G. Shen, Adv. Mater. 2014, 26, 4763.

[59] a) H. Xia, Y. S. Meng, G. Yuan, C. Cui, L. Lu, Electrochem. Solid-State Lett. 2012, 15, A60; b) V. Gupta, N. Miura, Mater. Lett. 2006, 60, 1466; c) T. Liu, L. Finn, M. Yu, H. Wang, T. Zhai, X. Lu, Y. Tong, Y. Li, Nano Lett. 2014, 14, 2522; d) M. Toupin, T. Brousse, D. Bélanger, Chem. Mater. 2004, 16, 3184.

[60] a) K. Y. T. Lee, H. H. Shi, K. Lian, H. E. Naguib, Smart Mater. Struct. 2015, 24, 115008; b) H. Lin, L. Li, J. Ren, Z. Cai, L. Qiu, Z. Yang, H. Peng, Sci. Rep. 2013, 3.

[61] a) Z.-F. Li, H. Zhang, Q. Liu, L. Sun, L. Stanciu, J. Xie, ACS Appl. Mater. Interfaces 2013, 5, 2685; b) K. H. An, W. S. Kim, Y. S. Park, J.-M. Moon, D. J. Bae, S. C. Lim, Y. S. Lee, Y. H. Lee, Adv. Funct. Mater. 2001, 11, 387.

[62] E. M. Ahmed, J. Adv. Res. 2013.

[63] a) Y. Shi, L. Pan, B. Liu, Y. Wang, Y. Cui, Z. Bao, G. Yu, J. Mater. Chem. A 2014, 2, 6086; b) Y. Xu, Z. Lin, X. Huang, Y. Wang, Y. Huang, X. Duan, Adv. Mater. 2013, 25, 5779; c) L. Zhang, G. Shi, J. Phys. Chem. C 2011, 115, 17206; d) G.-P. Hao, F. Hippauf, M. Oschatz, F. M. Wisser, A. Leifert, W. Nickel, N. Mohamed-Noriega, Z. Zheng, S. Kaskel, ACS Nano 2014, 8, 7138.

[64] Y. Xu, Z. Lin, X. Huang, Y. Liu, Y. Huang, X. Duan, ACS Nano 2013, 7, 4042.

[65] S. Ghosh, O. Inganäs, Adv. Mater. 1999, 11, 1214.

[66] Y. Xu, K. Sheng, C. Li, G. Shi, ACS Nano 2010, 4, 4324.

[67] P. Chen, J.-J. Yang, S.-S. Li, Z. Wang, T.-Y. Xiao, Y.-H. Qian, S.-H. Yu, Nano Energy 2013, 2, 249.

[68] J. Chen, K. Sheng, P. Luo, C. Li, G. Shi, Adv. Mater. 2012, 24, 4569.

[69] S. Chen, J. Duan, Y. Tang, S. Zhang Qiao, Chem. Eur. J. 2013, 19, 7118.

[70] F. Xiao, S. Yang, Z. Zhang, H. Liu, J. Xiao, L. Wan, J. Luo, S. Wang, Y. Liu, Sci. Rep. 2015, 5.

[71] J. Zang, C. Cao, Y. Feng, J. Liu, X. Zhao, Sci. Rep. 2014, 4.

[72] M. Lee, B. H. Wee, J. D. Hong, Adv. Energy Mater. 2015, 5.

[73] D. Sun, X. Yan, J. Lang, Q. Xue, J. Power Sources 2013, $222,52$.

[74] X. Zhao, C. M. Hayner, M. C. Kung, H. H. Kung, ACS Nano 2011, 5, 8739.

[75] G. Wang, X. Sun, F. Lu, H. Sun, M. Yu, W. Jiang, C. Liu, J. Lian, Small 2012, 8, 452.

[76] H. Chen, M. B. Müller, K. J. Gilmore, G. G. Wallace, D. Li, Adv. Mater. 2008, 20, 3557.

[77] W.-w. Liu, X.-b. Yan, J.-w. Lang, C. Peng, Q.-j. Xue, J. Mater. Chem. 2012, 22, 17245.

[78] J. Xu, D. Wang, L. Fan, Y. Yuan, W. Wei, R. Liu, S. Gu, W. Xu, Org. Electron. 2015, 26, 292.

[79] X. Zang, X. Li, M. Zhu, X. Li, Z. Zhen, Y. He, K. Wang, J. 
Wei, F. Kang, H. Zhu, Nanoscale 2015, 7, 7318.

[80] Y. Meng, K. Wang, Y. Zhang, Z. Wei, Adv. Mater. 2013, 25, 6985.

[81] S. Chabi, C. Peng, Z. Yang, Y. Xia, Y. Zhu, RSC Adv. 2015, 5, 3999.

[82] a) W. Wang, S. Guo, M. Penchev, I. Ruiz, K. N. Bozhilov, D. Yan, M. Ozkan, C. S. Ozkan, Nano Energy 2013, 2, 294; b) X. Dong, Y. Ma, G. Zhu, Y. Huang, J. Wang, M. B. Chan-Park, L. Wang, W. Huang, P. Chen, J. Mater. Chem. 2012, 22, 17044.

[83] C. Xu, T. Xue, J. Guo, Q. Qin, S. Wu, H. Song, H. Xie, J. Appl. Phys. 2015, 117, 164301.

[84] T. Choi, S. J. Kim, S. Park, T. Y. Hwang, Y. Jeon, B. H. Hong, Nanoscale 2015, 7, 7138.

[85] a) H.-J. Choi, S.-M. Jung, J.-M. Seo, D. W. Chang, L. Dai, J.-B. Baek, Nano Energy 2012, 1, 534; b) M. F. El-Kady, R. B. Kaner, Nat. Commun. 2013, 4, 1475; c) C. Liu, Z. Yu, D. Neff, A. Zhamu, B. Z. Jang, Nano Lett. 2010, 10, 4863.

[86] S. Xin, Y.-G. Guo, L.-J. Wan, Acc. Chem. Res. 2012, 45, 1759. 